\title{
Simplicity in the Structure of QED and Gravity Amplitudes
}

\author{
Simon Badger \\ Deutches Elektronen-Synchrotron DESY, \\ Platanenallee, 6, D-15738 Zeuthen, Germany \\ simon.badger@desy.de

\section{N. E. J. Bjerrum-Bohr} \\ School of Natural Sciences, \\ Institute for Advanced Study, \\ Einstein Drive, Princeton, \\ NJ 08540, USA \\ bjbohr@ias.edu

\section{Pierre Vanhove} \\ Institut des Hautes Etudes Scientifiques \\ Le-Bois-Marie \\ F-91440 Bures-sur-Yvette, France \\ and \\ Institut de Physique Théorique, \\ CEA, IPhT, F-91191 Gif-sur-Yvette, France \\ CNRS, URA 2306, F-91191 Gif-sur-Yvette, France \\ pierre.vanhove@cea.fr
}

ABSTRACT: We investigate generic properties of one-loop amplitudes in unordered gauge theories in four dimensions. For such theories the organisation of amplitudes in manifestly crossing symmetric expressions poses restrictions on their structure and results in remarkable cancellations. We show that one-loop multi-photon amplitudes in QED with at least eight external photons are given only by scalar box integral functions. This QED 'no-triangle' property is true for all helicity configurations and has similarities to the "notriangle' property found in the case of maximal $\mathcal{N}=8$ supergravity. Results are derived both via a world-line formalism as well as using on-shell unitarity methods. We show that the simple structure of the loop amplitude originates from the extremely good BCFW scaling behaviour of the QED tree-amplitude.

KEYWORDs: Amplitudes in fields theories, QED, Quantum gravity. 


\section{Contents}

1. Introduction 2

2. One-loop amplitudes in the world-line approach 3

2.1 One-loop amplitudes in colourless gauge theories 3

2.2 The reduction formulæ 8

2.3 Reduction of unordered one-loop amplitudes 9

3. Multi-photon amplitudes in QED with the unitarity method 10

3.1 The one-loop multi-photon amplitude 10

3.2 The tree-level amplitudes 12

3.2.1 The fermionic tree-level amplitudes 12

3.2.2 Massive scalar tree amplitudes 14

3.3 BCFW shifts and large momentum scaling at tree-level 15

3.3.1 Large- $z$ scaling for the massless tree amplitudes 15

3.3.2 Large- $z$ scaling of the massive scalar tree amplitudes 16

3.3.3 Origin of the improved BCFW scaling behaviour 17

3.4 One-loop structure from large $z$ momentum scaling 20

3.4.1 Vanishing of triangle coefficients 20

3.4.2 Vanishing of bubble coefficients 21

3.4.3 Vanishing of rational terms 21

$3.5 \quad$ No-triangle property of one-loop photon amplitudes 21

3.6 Universal ultra-violet and infra-red pole structure 22

3.6.1 The $n$-photon MHV Amplitude 23

4. Conclusions 24

A. Helicity formalism conventions 26

B. Proof of the improved large $z$ behaviour of the $e^{-} e^{+} \rightarrow n \gamma$ tree-amplitude 27

G. Cut analysis of one-loop $n$-photon integral coefficients 29

C.1 Absence of triangles in amplitudes with $n>6$

C.2 Absence of bubbles in amplitudes with $n>4$

C.3 Absence of rational terms 32

D. The scalar box integral functions 36 


\section{Introduction}

Powerful methods based on on-shell unitarity have led to the discovery of remarkable simplifications in analytic expressions of perturbative amplitudes in gauge theory [1] and gravity [2-7]. At one-loop order in four dimensions all $n$-point amplitudes can be expanded in a set of basis functions consisting of scalar box, triangle, and bubble integrals and possibly rational polynomial functions $[8,9]$. This is a consequence of the kinematic restrictions induced by working with four-dimensional momenta and the observation that amplitude expressions containing tensor integrals can be reduced to scalar integrals, although through extensive and cumbersome algebraic manipulations [8,10-12]. Generalisations to higher loop amplitudes are more complicated since a generic basis of integral functions is not known for an arbitrary number of legs. This persists even in relatively simple examples, such as in the planar limit of $\mathcal{N}=4$ super Yang-Mills where dual conformal symmetry restricts the form of amplitudes. Crossing symmetry in colourless theories require that both planar and non-planar integrals are present in the amplitude. This makes the construction of a basis of integral functions that captures the ultra-violet and infra-red behaviour of higher loop amplitudes more elusive [13-16]. Nevertheless, constraints from supersymmetry [17], string theory duality arguments $[18,19]$ and the field theory computations of [20-22] have explicitly shown that multi-loop amplitudes in $\mathcal{N}=8$ supergravity have much simpler forms than one would expect from a Lagrangian perspective.

In the case of colourless gauge theories the summation over all orderings of external legs (this includes both planar and non-planar contributions) leads to extra important cancellations in the amplitude. At one-loop order such cancellations lead to the 'no-triangle' property $[4,6,23-25]$ of $\mathcal{N}=8$ supergravity. We will show in this paper that for one-loop multi-photon amplitudes with $n \geq 8$ legs we have a similar 'no-triangle' property.

Recently, it has been shown in [23] that the world-line formalism (a.k.a. the 'string based method' for field theory amplitude computations [26-30]) is particularly well suited for exhibiting the cancellations coming from the summation over the various orderings of colourless gauge theories at one-loop order. The higher-loop extension of this formalism [30-33] presents a possible dimension independent framework for investigations of the improved ultra-violet behaviour of maximal $\mathcal{N}=8$ supergravity $[6,7,17-21,34]$. The power of the world-line formalism has been demonstrated in the recent study [34] of the two-loop supergravity four-graviton $\mathcal{N}=8$ amplitude given in ref. [35].

The unordered cancellations featured in gravity theories are also present in one-loop multi-photon amplitudes in QED and Super-QED but in a simpler framework. One-loop multi-photon amplitudes with $n$ external legs have naïvely $n$ powers of loop momenta. This indicates that under $n$ steps of Passarino-Veltman reductions $[8,10-12]$ the amplitude would, a priori, contain scalar box, triangle and bubble integrals and may also contain rational polynomial (non-logarithmic) contributions. However, explicit computations show that the true structure is somewhat simpler.

For the case of multi-photon massless QED amplitudes we can explain the discrepancy between the naïve power counting and explicit results using reduction formulæ for unordered amplitudes [23], that were derived using the world-line formalism. We will di- 
rectly show that these reduction formulæ imply that the four-photon one-loop amplitude reduces down to scalar box triangle and bubble integral functions together with rational terms. For the six-photon amplitude that it reduces to box and three-mass scalar triangle integral functions. For multi-photon amplitudes beyond six-point we have a reduction down to scalar box integral functions only. This box structure only of the amplitude for $n \geq 8$ external photons is true for all helicity configurations but the precise expansion of the amplitude in terms of scalar box integral functions depends on the choice of helicity for the external states. Our observations are in complete correspondence with the recent direct evaluation of the one-loop six-photon amplitude in $[36,37]$. An earlier evaluation of the multi-photon MHV amplitude by Mahlon [38] showed that in this case the amplitude contains only massive box integral contributions. This sheds further light on how considering expressions for unordered amplitudes with full crossing symmetry leads to a surprising simplicity for amplitudes.

The structure of the paper is as follows. In section 2 we review in details the recent results for the reductions of unordered integral functions at one-loop. On general grounds we will then investigate via a string-based formalism how reduction formulæ can be induced by invariance of amplitudes under gauge transformations $[23,24,39]$. In section 2.2 we will re-derive the reduction formulæ in ref. [23] and discuss their consequences for unordered amplitudes. In section 3 we confirm the results obtained using the world-line method with on-shell unitarity methods. Many advances in generalised unitarity techniques have been made recently both in the context of analytic computations [40-43] and for numerical evaluations [44-46]. Using such analytic methods that exploit complex analysis and factorisation properties $[43,47]$ we show that the 'no triangle' property for multi-photon amplitudes follows from the behaviour of the tree amplitudes as the momentum flowing in the cut becomes large. The appendices contains the technical details on the evaluation of the cut amplitudes.

\section{One-loop amplitudes in the world-line approach}

In this section we will describe the world-line approach of ref. [23] for analysing the structure of multi-leg one-loop amplitudes for colourless gauge theories. One important property of colourless gauge theories is that the tensorial structure associated with each different ordering of the external legs is the same. This leads to cancellations that are not manifestly featured in the ordered amplitudes. Within the traditional Feynman graph approach this fact is difficult to implement but in the world-line approach it is particularly transparent and leads to the specific set of reduction formulæ derived in ref. [23].

\subsection{One-loop amplitudes in colourless gauge theories}

The generic structure of colourless gauge theory amplitudes at one-loop, e.g., in QED or gravity can be given by the following expression based on a Schwinger proper-time representation of the one-loop amplitude [26-30]

$$
\mathcal{A}_{n}^{\text {one-loop }}=\int_{0}^{\infty} \frac{d T}{T} T^{-D / 2+n} \int_{0}^{1} d^{n-1} \nu \mathcal{P}\left(h_{i}, k_{i} ; \nu_{i}\right) \exp \left(-T Q_{n}\right) .
$$


In this equation $T$ is the one-loop proper-time and $\nu_{i}$ are Feynman parameters associated with the external states of the amplitude. These are integrated over the range $[0,1]$ with the following measure of integration

$$
\int_{0}^{1} d^{n-1} \nu \equiv \prod_{i=1}^{n} \int_{0}^{1} d \nu_{i} \frac{1}{n} \sum_{j=1}^{n} \delta\left(\nu_{j}=1\right) .
$$

We will use a symmetrised delta-function to fix the translational invariance in the loop amplitude. The quantity $Q_{n}$ is defined by

$$
Q_{n} \equiv \sum_{1 \leq i<j \leq n}\left(k_{i} \cdot k_{j}\right) G_{B}\left(\nu_{i}-\nu_{j}\right) .
$$

The one-loop scalar world-line Green function $G_{B}(x)$ is defined by

$$
G_{B}(x)=x^{2}-|x|,
$$

and is the solution to the one-dimensional Poisson equation

$$
\partial_{x}^{2} G_{B}(x)=\delta(x) .
$$

$G_{B}(x)$ is the infinite tension, $\alpha^{\prime} \rightarrow 0$, limit of the corresponding bosonic string correlator (see [27-30] for a justification of these rules)

$$
G_{B}(\nu)=-\frac{1}{6}+\frac{1}{D} \lim _{\alpha^{\prime} \rightarrow 0} \eta_{m n}\left\langle x^{m}(\nu) x^{n}(0)\right\rangle=\sum_{n \in \mathbb{Z} \backslash\{0\}} \frac{1}{n^{2}} e^{2 i \pi n \nu}-\frac{1}{6} .
$$

The constant $1 / 6$ in the above equation does not contribute to the on-shell amplitudes because of momentum conservation. See refs. [30-33] for a generalisation of the world-line formalism to higher-loop amplitudes.

We will also introduce the fermionic Green function

$$
G_{F}(x)=\operatorname{sign}(x) .
$$

$G_{F}(x)$ is defined as the infinite tension, $\alpha^{\prime} \rightarrow 0$, limit of the world-line correlator for fermions $\psi^{m}(\nu)$ with the anti-periodic boundary conditions $\psi^{m}(\nu+1)=-\psi^{m}(\nu)$. The correlator $G_{F}(x)$ can be expressed as (see $[27,28,30]$ for a justification of these rules)

$$
G_{F}(\nu)=\frac{1}{D} \lim _{\alpha^{\prime} \rightarrow 0} \eta_{m n}\left\langle\psi^{m}(\nu) \psi^{n}(0)\right\rangle_{A}=2 \sum_{n \in \mathbb{Z}+\frac{1}{2}} \frac{e^{2 i \pi n \nu}}{n} .
$$

The representation (2.1) of the one-loop amplitude can be obtained by considering a Schwinger representation of the corresponding Feynman integrals. For instance by an exponentiation of the propagators of the loop amplitude with the external states arranged in the order $\{1,2, \ldots, n\}$ along the loop one can write

$$
\begin{aligned}
\prod_{i=1}^{n} \frac{1}{\left(\ell-k_{1 \cdots i}\right)^{2}} & =\int_{0}^{\infty} \prod_{i=1}^{n} d \alpha_{i} \exp \left(-\sum_{i=1}^{n} \alpha_{i}\left(\ell-k_{1 \cdots i}\right)^{2}\right) \\
& =\int_{0}^{T} d T T^{1-n} \int_{0}^{1} \prod_{i=1}^{n-1} d a_{i} \exp \left(-T\left(\ell-K_{[n]}\right)^{2}-T Q_{n}\right)
\end{aligned}
$$


where $k_{1 \cdots i}=k_{1}+k_{2}+\cdots+k_{i}$ and the rescaled Schwinger parameters $a_{i}=\alpha_{i} / T$ are related to the $\nu_{i}$ in eq. (2.1) by

$$
\nu_{i}=\sum_{j=1}^{i} a_{j}
$$

As in [23] we use $\sigma$ to denote a given ordering. ( $\sigma$ is defined as a given permutation of the $n$ external legs $\left.\left\{k_{\sigma(1)}, \ldots, k_{\sigma(n)}\right\}\right)$. In this notation the mapping between the $\nu_{i}$ and $a_{i}$ variables is given by

$$
\nu_{i}=\sum_{j=1}^{i} a_{\sigma(j)} .
$$

In the above representation one sees that the loop momentum is given by the total inflow of external momenta

$$
K_{[n]}=\sum_{j=1}^{n} k_{j} \nu_{j}
$$

In this representation a power of loop momentum $\ell \cdot k_{i}$ appearing in the numerator of the Feynman integral has the following representation

$$
2 k_{i} \cdot K_{[n]}=2 \sum_{j=1}^{n}\left(k_{i} \cdot k_{j}\right) \nu_{j}=-\partial_{\nu_{i}} Q_{n}+2 \sum_{j=1}^{n}\left(k_{i} \cdot k_{j}\right) G_{F}\left(\nu_{i}-\nu_{j}\right) .
$$

This shows that in the world-line representation the powers of loop momenta in the amplitude are counted by the first derivative $\partial_{\nu_{i}} Q_{n}$. Following the strategy defined in eq. $[23,24]$ we expand the polarisations of the external states in a basis of independent momenta

$$
h_{i}=\sum_{j=1}^{n-1} c_{i}^{j} k_{j}+q^{\perp}
$$

Here $q^{\perp}$ is a vector orthogonal to the $(n-1)$ linearly independent external momenta. For an amplitude with $n>4$ external legs the momentum $q^{\perp}$ is only needed in dimensions $D>4$. One employs an identical definition for the $\bar{h}_{i}$ polarisations. Using the relation (2.3) one easily derives that

$$
2 h_{i} \cdot K_{[n]}=2 \sum_{r=1}^{n-1} c_{i}^{r} k_{r} \cdot K_{[n]}=\sum_{r=1}^{n-1} c_{i}^{r}\left[-\partial_{\nu_{r}} Q_{n}+\sum_{j=1}^{n}\left(k_{r} \cdot k_{j}\right) G_{F}\left(\nu_{r}-\nu_{j}\right)\right] .
$$

It should be noticed as well that the second derivative on $Q_{n}$ is given by

$$
\begin{aligned}
\partial_{\nu_{i}} \partial_{\nu_{j}} Q_{n} & =\left(k_{i} \cdot k_{j}\right) \partial_{\nu_{i}} \partial_{\nu_{j}} G_{B}\left(\nu_{i}-\nu_{j}\right) & & \text { no sum over } i, j \\
& =2\left(k_{i} \cdot k_{j}\right)\left(\delta\left(\nu_{i}-\nu_{j}\right)-1\right) & & \text { no sum over } i, j,
\end{aligned}
$$

and does not contain any powers of loop momenta. The delta-function $\delta\left(\nu_{i}-\nu_{j}\right)$ in the above expression pinches two of the external legs. The constant arises from the zero-mode contribution to the world-line Green function. 
The dependence on the external polarisations $h_{i}$ and momenta $k_{i}$ with $1 \leq i \leq n$ is given by the function $\mathcal{P}\left(h_{i}, k_{i} ; \nu_{i}\right)$ which for the massless QED amplitude takes the form

$$
\mathcal{P}\left(h_{i}, k_{i} ; \nu_{i}\right)=\left.\prod_{i=1}^{n} \int d \theta_{i} \exp (\mathcal{F})\right|_{\text {linear in } h_{i}} .
$$

Here $\theta_{i}^{\alpha}$ are $n$ Grassmann variables that we discuss below, and one has to keep only the terms linear in each of the polarisations $h_{i}$ of the external states (with $1 \leq i \leq n$ ). The factor $\mathcal{F}$ is defined by

$$
\begin{aligned}
\mathcal{F} & =\frac{1}{2 T} \sum_{i \neq j}\left(h_{i} \cdot h_{j}\right) \theta_{i} \theta_{j} \partial_{\nu_{i}} \partial_{\nu_{j}} G_{B}\left(\nu_{i}-\nu_{j}\right)+\frac{i}{2} \sum_{i \neq j}\left(k_{i} \cdot h_{j} \theta_{i}-k_{j} \cdot h_{i} \theta_{j}\right) \partial_{i} G_{B}\left(\nu_{i}-\nu_{j}\right) \\
& +\frac{1}{2} \sum_{i \neq j}\left(h_{i} \cdot h_{j}\right) G_{F}\left(\nu_{i}-\nu_{j}\right)-\frac{i}{2} \sum_{i \neq j}\left(k_{i} \cdot h_{j} \theta_{j}-k_{j} \cdot h_{i} \theta_{i}\right) G_{F}\left(\nu_{i}-\nu_{j}\right) \\
& +\frac{1}{2} \sum_{i \neq j} \theta_{i} \theta_{j}\left(k_{i} \cdot k_{j}\right) G_{F}\left(\nu_{i}-\nu_{j}\right) .
\end{aligned}
$$

This factor is derived by considering the correlation function of $n$ vertex operators for a $U(1)$ gauge boson

$$
V_{i}=\left(h_{i} \cdot \partial x+i k_{i} \cdot \psi h_{i} \cdot \psi\right) e^{i k_{i} \cdot x}
$$

as

$$
\left\langle V_{1} \cdots V_{n}\right\rangle=\exp (\mathcal{F}) \exp \left(-T Q_{n}\right)
$$

The expression (2.18) has been written introducing the fermionic variable $\theta_{i}$

$$
\begin{aligned}
V_{i} & =\int d \theta_{i} h_{i} \cdot D X e^{i k_{i} \cdot X}=\left.\int d \theta_{i} \exp \left(h_{i} \cdot D X+i k_{i} \cdot x\right)\right|_{\text {linear in } h_{i}} \\
& =\left.\int d \theta_{i} \exp \left(\theta_{i}\left(h_{i} \cdot \partial x\right)+h_{i} \cdot \psi+\theta_{i}\left(i k_{i} \cdot \psi\right)+i k_{i} \cdot x\right)\right|_{\text {linear in } h_{i}},
\end{aligned}
$$

where $\theta$ is a fermionic variable in the $N=1$ world-line formalism and $X$ is a superfield $X^{m}=x^{m}+\theta \psi^{m}$ with the fermionic derivative $D=\partial_{\theta}-\theta \partial_{\nu}$ (see ref. [30,33,48] for further details). The contractions between the world-line fields are done using the correlators in eqs. (2.6) and (2.8).

Using the relations in eqs. 2.13), (2.14) and (2.15) one can show [23] that the generic form of a QED amplitude is given by the sum of unordered $n$-point integrals $\mathcal{I}_{n}\left[\mathrm{I}_{r}, \mathrm{~J}_{s}\right]$ evaluated in $D$ dimensions where the positions of the external states are freely integrated over the loop

$$
\mathcal{I}_{n}\left[\mathrm{I}_{r}, \mathrm{~J}_{s}\right]=\int_{0}^{1} d^{n-1} \nu Q_{n}^{D / 2-n} \prod_{i \in \mathrm{I}_{r}} \partial_{i} Q_{n} \prod_{x \in \mathrm{J}_{s}} G_{F}(x),
$$

with gauge invariant tensorial coefficients $t_{r, s}^{l}$ built from the external momenta and polarisations

$$
\mathcal{A}_{n}^{\text {one-loop }}=\sum_{u=0}^{n / 2} \sum_{l=0}^{u} \sum_{r+s+2 l=n} t_{r, s}^{l} \mathcal{I}_{n-l}^{[D+2(u-l)]}\left[\mathrm{I}_{r}, \mathrm{~J}_{s}\right] \text {. }
$$


Here $\mathrm{I}_{r}=\left\{i_{1}, \ldots, i_{r}\right\}$ is a set of $r$ indices of the external states, and $\mathrm{J}_{s}=\left\{\nu_{j_{1}}-\nu_{l_{1}}, \ldots, \nu_{j_{s}}-\right.$ $\left.\nu_{l_{s}}\right\}$ is a set of $s$ differences of the positions of the external states. Because of the zero-mode contributions to the propagator $G_{B}(x)$ (see eq. (2.16)) the expression involves integrals that are evaluated in a dimension different from $D$ that we denote by introducing a superscript indicating the dimension where the integral are evaluated, e.g., $\mathcal{I}_{n}^{[D+2 u]}\left[\mathrm{I}_{r}, \mathrm{~J}_{s}\right]$.

Using the relation in eq. 2.13) one can deduce that the number of loop momenta in a one-loop $n$-photon amplitude in QED (given by eq. (2.23)) satisfies the constraint

$$
r+s \leq n .
$$

We will also quickly review the result for the graviton amplitude as given in [23]. The generic form of an $n$-graviton amplitude in $\mathcal{N}=8$ supergravity is given by

$$
\mathcal{M}_{n}^{\text {one-loop }}=\sum_{u=0}^{n / 2} \sum_{l=0}^{u} \sum_{r+s+2 l=2 n-8} t_{r, s}^{l} \mathcal{I}_{n-l}^{[D+2(u-l)]}\left[\mathrm{I}_{r}, \mathrm{~J}_{s}\right] .
$$

This expression displays that the $n$-graviton one-loop amplitude has at most $2 n-8$ powers of the loop momentum and satisfies,

$$
r+s \leq 2 n-8
$$

The upper bound arises because the two-derivative nature of the gravitational vertex imply that the amplitude has at most $2 n$ powers of loop momentum and eight powers of the loop momentum are cancelled by the integration over the sixteen fermionic zero modes.

Before we close this section we would like to make a few remarks:

- In computations of colourless one-loop amplitudes for gauge theories in the world-line approach all different orderings of legs in eq. (2.1) have the same tensorial structure. This particular point makes colourless gauge theory amplitudes special and makes it possible $[23,24]$ to reduce the amplitudes to a form consisting of a sum of $\mathcal{I}_{n}^{[D+2 u]}\left[\mathrm{I}_{r}, \mathrm{~J}_{s}\right]$ integrals as given in eqs. (2.23) and (2.25).

- Expressions for QED and supergravity amplitudes in eqs. (2.23) and (2.25) contain non-analytic functions featuring absolute numerical values of differences between the $\nu$ variables as well as sign functions in the definition of $G_{F}(x)$ in eq. (2.7). These non-analytic functions are lifted when the loop integral is formally evaluated and that splits the integrals up into sums of different regions of analyticity of the amplitude. The sum over these different regions of analyticity is in direct correspondence with the sum over different physical orderings of the amplitude.

- The representation of the one-loop amplitude in massless QED and supergravity given in (2.1) is readily obtained by considering the infinite tension limit $\alpha^{\prime} \rightarrow 0$ of the corresponding closed one-loop amplitude. No massive string modes play a role in these computations [27-30]. An extension of this world-line construction to higherloop amplitudes in $\mathcal{N}=8$ supergravity would give a field theoretic justification of the behaviour of the multi-loop four-graviton amplitude derived using string theory [17] and dualities in $[18,19,34]$. 


\subsection{The reduction formulæ}

The integrals forming the building blocks of the QED amplitude in eq. (2.23) and the supergravity amplitudes in eq. (2.25) satisfy new types of reduction formulæ that were derived in [23]. We will review these in this section.

The basic building block of colourless gauge theories are the unordered scalar $n$-point integrals

$$
\mathcal{I}_{n}\left[\mathrm{I}_{r+1}\right]=\int_{0}^{1} d^{n-1} \nu Q_{n}^{D / 2-n} \prod_{i \in \mathrm{I}_{r+1}} \partial_{i} Q_{n} .
$$

Here $\mathrm{I}_{r+1} \equiv\left\{i_{1}, \ldots, i_{r+1}\right\}$ is a set of $r+1$ distinct indices taking values in $\{1, \ldots, n\}$. It was shown in [23] that, by integration by parts, these integrals satisfy the reduction formulæ

$$
\begin{aligned}
& \mathcal{I}_{n}\left[\mathrm{I}_{r+1}\right]= \\
& \frac{2}{D / 2-n+1}\left[\sum_{j \in \mathrm{I}_{r-m+1}}\left(k_{i_{r+1}} \cdot k_{j}\right)\left(-\mathcal{I}_{n-1}^{\left(i_{r+1} j\right)}\left[\mathrm{I}_{r-1}^{(j)}\right]+\mathcal{I}_{n}^{[D+2]}\left[\mathrm{I}_{r-1}^{(j)}\right]\right)\right. \\
& \left.+(m-1) \sum_{s=1}^{n}\left(k_{i_{r+1}} \cdot k_{s}\right) \mathcal{I}_{n-1}^{\left(i_{r+1} s\right)}\left[\mathrm{I}_{r-1}^{(r+1)}\right]\right] .
\end{aligned}
$$

We see that $\mathcal{I}_{n}\left[\mathrm{I}_{r+1}\right]$ can be expressed as a sum of the dimension shifted integrals $\mathcal{I}_{n}^{[D+2]}\left[\mathrm{I}_{r-1}\right]$ and the one-mass $(n-1)$-point integrals

$$
\mathcal{I}_{n-1}^{(i j)}\left[\mathrm{I}_{r-1}^{(j)}\right] \equiv \int_{0}^{1} d^{n-1} \nu Q_{n}^{D / 2-n} \delta\left(\nu_{i}-\nu_{j}\right) \prod_{s \in \mathrm{I}_{r-1}^{(j)}} \partial_{\nu_{s}} Q_{n} .
$$

Integrals with more than one mass are defined in the same way with several delta function insertions. The boundary term is vanishing because of the 1-periodicity of $Q_{n}$ in each of the $\nu_{i}$ variables, $Q_{n}\left(\nu_{1}, \ldots, \nu_{i}+1, \ldots\right)=Q_{n}\left(\nu_{1}, \ldots, \nu_{i}, \ldots\right)$ since $G_{B}(1-x)=G_{B}(x)$ for $0 \leq x \leq 1$ and $G_{B}(0)=G_{B}(1)=0$.

As in [23] the rule eq. (2.28) can be summarised as

$$
\mathcal{I}_{n}\left[\left(\partial Q_{n}\right)^{r}\right] \rightsquigarrow \mathcal{I}_{n-1}^{\text {mass }}\left[\left(\partial Q_{n}\right)^{r-2}\right]+\mathcal{I}_{n}^{[D+2]}\left[\left(\partial Q_{n}\right)^{r-2}\right],
$$

where $\mathcal{I}_{n}^{\text {mass }}$ denotes a massive $n$-point integral. Using the relations (2.13) and 2.15) between the loop momenta and the derivative of $Q_{n}, \ell \sim \partial_{\nu} Q_{n}$, this relation implies that two powers of loop momenta $\ell$ are cancelled at each step

$$
\mathcal{I}_{n}\left[\ell^{r}\right] \rightsquigarrow \mathcal{I}_{n-1}^{\operatorname{mass}}\left[\ell^{r-2}\right]+\mathcal{I}_{n}^{[D+2]}\left[\ell^{r-2}\right] .
$$

When some factors of $G_{F}(x)$ are present in the integrand we have to distinguish between the following cases

$\triangleright$ If all the $i \in \mathrm{I}_{r}$ are such that $\nu_{i}$ is not an argument of $G_{F}(x)$ for any $x \in \mathrm{J}_{s}$, then the same manipulations leading to eq. (2.28) apply with no changes.

$\triangleright$ If $i_{r+1}$ has multiplicity one in $\mathrm{I}_{r+1}=\mathrm{I}_{r} \cup\left\{i_{r+1}\right\}$ with $i_{r+1} \notin \mathrm{I}_{r}$ and $\mathrm{J}_{1}=\left\{\nu_{i_{r+1}}-\nu_{j}\right\}$ then 


$$
\mathcal{I}_{n}\left[\mathrm{I}_{r+1}, \mathrm{~J}_{1}\right]=\frac{1}{D / 2-n+1} \int_{0}^{1} d^{n-1} \nu \partial_{\nu_{i_{r+1}}} Q_{n}^{D / 2-n+1} G_{F}\left(\nu_{i_{r+1}}-\nu_{j}\right) \prod_{i \in \mathrm{I}_{r}} \partial_{i} Q_{n} .
$$

This leads, after integration by parts, to

$$
\begin{aligned}
\mathcal{I}_{n}\left[\mathrm{I}_{r+1}, \mathrm{~J}_{1}\right]= & \frac{2}{D / 2-n+1} \times \\
& {\left[\sum_{j \in \mathrm{I}_{r}}\left(k_{i_{r+1}} \cdot k_{j}\right)\left(-\mathcal{I}_{n-1}^{\left(i_{r+1} j\right)}\left[\mathrm{I}_{r-1}^{(j)}, \mathrm{J}_{1}\right]+\mathcal{I}_{n}^{[D+2]}\left[\mathrm{I}_{r-1}^{(j)}, \mathrm{J}_{1}\right]\right)\right.} \\
& \left.+\left((n-1) \mathcal{I}_{n-1}^{\left(i_{r+1} j\right)}\left[\mathrm{I}_{r}\right]-\sum_{l=1}^{n} \mathcal{I}_{n}^{\left(i_{k+1} l\right)}\left[\mathrm{I}_{r}^{(r+1)}\right]\right)\right] .
\end{aligned}
$$

This expression is easily generalised to other cases, with higher multiplicity of $i_{k+1}$ and with additional $G_{F}$ contributions.

As in [23] this rule can be summarised by

$$
\mathcal{I}_{n}\left[\left(\partial Q_{n}\right)^{r}, G_{F}\right] \rightsquigarrow \mathcal{I}_{n-1}^{\text {mass }}\left[\left(\partial Q_{n}\right)^{r-1}\right]+\mathcal{I}_{n-1}^{\text {mass }}\left[\left(\partial Q_{n}\right)^{r-2}, G_{F}\right]+\mathcal{I}_{n}^{[D+2]}\left[\left(\partial Q_{n}\right)^{r-2}, G_{F}\right] .
$$

Using the relations (2.13) and (2.15) between the loop momentum $\ell$ and the first derivative of $Q_{n}, \ell \sim \partial_{\nu} Q_{n}$, this relation can be rewritten as

$$
\mathcal{I}_{n}\left[\ell^{r}, G_{F}\right] \rightsquigarrow \mathcal{I}_{n-1}^{\text {mass }}\left[\ell^{r-1}\right]+\mathcal{I}_{n-1}^{\operatorname{mass}}\left[\ell^{r-2}, G_{F}\right]+\mathcal{I}_{n}^{[D+2]}\left[\ell^{r-2}, G_{F}\right] .
$$

\subsection{Reduction of unordered one-loop amplitudes}

As was explained in ref. [23] because one-loop amplitudes of $\mathcal{N}=8$ supergravity takes the symbolic form

$$
\mathcal{M}_{n ; 1}=\sum_{\substack{r+s+u=2 n-\mathcal{N} \\ 0 \leq u \leq n}} \sum_{l=0}^{u} t_{r, s}^{l} \mathcal{I}_{n-l}^{[D+2(u-l)]}\left[\mathrm{I}_{r}, \mathrm{~J}_{s}\right]
$$

and due to the the constraint $r+s \leq 2 n-8$, all amplitudes can eventually be reduced to scalar box integral functions. In this section we apply the reduction formulæ of eqs. (2.30) and (2.34) to the QED amplitude (2.23) and repeat our analysis in ref. [23] for the QED case.

The structure of the derivative structure of the cubic $q \bar{q} \gamma$ vertex implies that an $n$ photon one-loop amplitude has at most $n$ powers of loop momenta. The generic form of the QED amplitude is given by

$$
\mathcal{A}_{n}^{\text {one-loop }}=\sum_{u=0}^{n / 2} \sum_{l=0}^{u} \sum_{r+s+2 l=n} t_{r, s}^{l} \mathcal{I}_{n-l}^{[D+2(u-l)]}\left[\mathrm{I}_{r}, \mathrm{~J}_{s}\right] .
$$

In QED we have the constraint

$$
r+s \leq n,
$$

in the decomposition of the amplitude. In fact because of Furry's theorem stated in eq. (3.5) the non-vanishing amplitudes will have an even number of external photon states $n=2 m$. 
We will comment more on this in section 3.1. Applying the reduction formulæ of the previous section to the contribution with the highest power of loop momenta $\mathcal{I}_{n}\left[I_{n}\right]$ will reduce it to $\mathcal{I}_{m}[\emptyset]$ after $n / 2=m$ steps of reductions plus a contribution from dimension shifted integrals. All other contributions with less powers of loop momenta, i.e., $r+s \leq n$ with $s \neq 0$, will reduce as well to $\mathcal{I}_{m}[\emptyset]$ plus the contribution from dimension shifted integrals. The dimension shifted integrals are of the type

$$
\mathcal{I}_{n+p}^{[4+2 p]}[\emptyset]=\int_{0}^{\infty} \frac{d^{4} \ell d^{2 p} \ell_{\perp}}{(2 \pi)^{4+2 p}} \prod_{i=1}^{n+p} \frac{1}{\left(\ell-k_{1}-\cdots-k_{i}\right)^{2}+\ell_{\perp}^{2}}
$$

with $D=4$ and $1 \leq p \leq n$. These integrals do not carry any ultra-violet nor infra-red divergences and have the special structure of the loop momentum being integrated in $4+2 p$ dimensions with $p \geq 1$ but with only four dimensional external momenta. The dimension shifted contributions are an artifact of the reduction formulæ and they do not contribute to the the total physical amplitudes as shown in [23].

From this analysis we can conclude that one-loop amplitudes with $n>4$ external photons do not contains any scalar bubble integrals. Amplitudes with $n>6$ external photons do not contain any scalar triangle integrals and will be completely specified by scalar box integrals. We will confirm these results directly via on-shell unitarity methods in the following sections.

\section{Multi-photon amplitudes in QED with the unitarity method}

In the previous section we analysed the structure of the $n$-photon one-loop amplitude in massless QED from a string based world line formalism. We showed that the amplitudes with $n>4$ external photons do not contains any scalar bubble integrals and that amplitudes with $n>6$ external photons do not contain any scalar triangle integrals and hence are completely specified by scalar box integrals.

These results are in agreement with the explicit evaluation of the one-loop four-photon amplitude in [38] and the six-photon amplitude computation in [36,37].

In this section we will consider multi-photon one-loop amplitudes in QED at the field theory level. The aim is to verify the above string-based arguments for the structure of the loop amplitude in QED. Looking at a simpler theory than gravity, QED, we hope to

shed light on how cancellations between various orderings in unordered field theories can take place.

\subsection{The one-loop multi-photon amplitude}

In this section we turn to the analysis of the photon scattering at one-loop in massless QED

$$
\gamma\left(k_{1}\right)+\cdots+\gamma\left(k_{n}\right) \rightarrow 0 .
$$

All photon lines are attached to the massless fermion loop. As for the case of the tree-level amplitude the one-loop amplitude can be written as a sum over all the orderings of the 


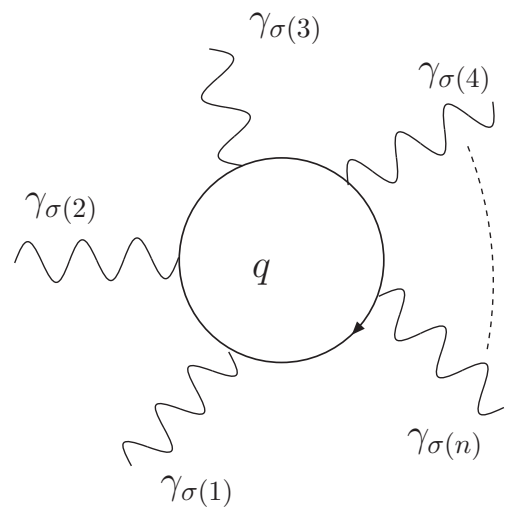

Figure 1: The one-loop $n$-photon amplitude in QED is the sum over all permutations of ordered photon lines attached to a fermion loop.

external photon lines

$$
A_{n ; \Phi}^{\text {one-loop }}\left(k_{1}, \ldots, k_{n}\right)=\sum_{\sigma \in \mathfrak{S}_{n}} \mathcal{A}_{n ; \Phi}^{\text {one-loop }}\left(k_{\sigma(1)}, \ldots, k_{\sigma(n)}\right) .
$$

Here $\mathcal{A}_{n ; \Phi}^{\text {one-loop }}\left(k_{\sigma(1)}, \ldots, k_{\sigma(n)}\right)$ is the ordered one-loop amplitude with $n$-photon lines attached to a fermion loop for $\Phi=q$ or a complex scalar for $\Phi=\varphi$.

We will split the cut analysis of the photon amplitude into two parts, one that can be determined from standard four-dimensional cuts and one involving a $D$-dimensional component that can be determined via massive cuts. We will write the full amplitude as:

$$
A_{n ; q}^{\text {one-loop }}\left(k_{1}, \ldots, k_{n}\right)=A_{n ; q}^{\text {one-loop }, C C}\left(k_{1}, \ldots, k_{n}\right)+R_{n}\left(k_{1}, \ldots, k_{n}\right) .
$$

Here the term $A_{n}^{\text {one-loop, } C C}$ contains all divergences and logarithmic terms and the term $R_{n}$ contains all remaining rational functions. The cut-constructible piece can be determined from cuts of photon amplitudes with a massless internal fermion line. By using the supersymmetric decomposition in terms of an $\mathcal{N}=1$ chiral multiplet and a scalar loop contribution,

$$
A_{n ; q}^{\text {one-loop }}\left(k_{1}, \ldots, k_{n}\right)=A_{n ; \mathcal{N}=1}^{\text {one-loop }}\left(k_{1}, \ldots, k_{n}\right)-A_{n ; \varphi}^{\text {one-loop }}\left(k_{1}, \ldots, k_{n}\right),
$$

it is clear that the rational terms originate from the scalar loop $A_{n ; \varphi}^{\text {one-loop }}\left(k_{1}, \ldots, k_{n}\right)$ since the $\mathcal{N}=1$ amplitude is cut-constructible in four dimensions [49]. We can therefore proceed to calculate these terms by computing cuts of the photon amplitude with a massive scalar loop. ${ }^{1}$

Before embarking on a detailed analysis of the structure of the multi-photon one-loop amplitudes in QED, we remark that because the coupling of a photon to a pair of fermions

\footnotetext{
${ }^{1}$ We note that it would also be possible to treat these contributions directly by using a massive internal fermion loop within the $D$-dimensional cutting method as demonstrated in reference [50]. However, we choose to compute the rational terms from the massive scalar loop since the cancellations from the orderings of the external legs in the tree-level amplitudes can be made more explicit.
} 
or complex scalar is odd under charge conjugation, the ordered amplitudes satisfy the following relation

$$
\mathcal{A}_{n ; \Phi}^{\text {one-loop }}\left(k_{1}, \ldots, k_{n}\right)=(-1)^{n} \mathcal{A}_{n ; \Phi}^{\text {one-loop }}\left(k_{n}, \ldots, k_{1}\right)
$$

The total amplitude summed over all orderings of the external photon lines will therefore vanish for an odd number of external states. This is the so-called Furry's theorem. It is valid for fermionic and scalar loop and for $\mathcal{N}=1$ super-QED amplitudes.

\subsection{The tree-level amplitudes}

Since the unitarity method constructs loop amplitudes from products of on-shell tree-level amplitudes, we will in this section first review the tree-level amplitudes needed to derive the one-loop photon amplitude.

\subsubsection{The fermionic tree-level amplitudes}

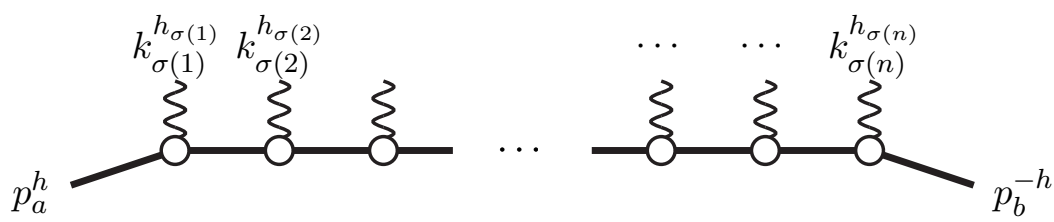

Figure 2: Tree-level $e^{+} e^{-}+n(\gamma) \rightarrow 0$ Feynman diagram for the ordering $\sigma \in \mathfrak{S}_{n}$. All the momenta are assumed to be incoming. The helicity of the fermions is given by $h= \pm \frac{1}{2}$.

The relevant tree amplitudes to the contribution of $A_{n ; q}^{\text {one-loop, } C C}$ coming from an internal massless fermion are those with photons coupling to a pair of massless fermions. Since this is an extremely simple process it is sufficient to use Feynman rules to write down the amplitude as a sum over $n$ factorial permutations,

$$
A_{n ; q}^{\text {tree }}\left(p_{a}^{h}, p_{b}^{-h} ; k_{1}, \ldots, k_{n}\right)=e^{n} \sum_{\sigma \in \mathfrak{S}_{n}} \mathcal{A}_{n ; q}^{\text {tree }}\left(p_{a}^{h}, p_{b}^{-h} ; k_{\sigma(1)}, \ldots, k_{\sigma(n)}\right) .
$$

We refer to [51] for the QED Feynman rules. All particles are considered to be incoming with momentum conservation defined by $p_{a}+p_{b}+\sum_{i=1}^{n} k_{i}=0$. We denote by the symbol $\mathfrak{S}_{n}$ the set of permutations of the $n$ objects and we denote a specific permutation by $\sigma \in \mathfrak{S}_{n}$. In the above we have represented the momenta of the fermion and anti-fermion by $p_{a}$ and $p_{b}$ respectively while the $n$ photons have been assigned momenta $k_{i}$ and helicities $h_{i}$. The ordered tree contribution, $\mathcal{A}^{\text {tree }}$, from an individual Feynman diagram can in all generality be written as

$$
\mathcal{A}_{n ; q}^{\text {tree }}\left(p_{a}^{h}, p_{b}^{-h} ; k_{\sigma(1)}, \ldots, k_{\sigma(n)}\right)=\bar{u}_{h}\left(p_{a}\right) \phi_{\sigma(1)} \frac{i \not q_{1}}{q_{1}^{2}} \phi_{\sigma(2)} \frac{i \not q_{2}}{q_{2}^{2}} \cdots \phi_{\sigma(n-1)} \frac{i \not q_{n-1}}{q_{n-1}^{2}} \phi_{\sigma(n)} u_{-h}\left(p_{b}\right) .
$$

Here $\not \gamma=\gamma^{\mu} v_{\mu}$ and $u_{2 h}(p)$ is the polarisation of the fermion with helicity $h= \pm \frac{1}{2}$. 
The polarisation of the $i^{\text {th }}$ photon is denoted by $\epsilon_{i}$, and $q_{i}$ is the momentum flowing between leg $i$ and $i+1$

$$
q_{i}=K_{\sigma(i)}+p_{a}, \quad \text { where } \quad K_{\sigma(i)} \equiv \sum_{r=1}^{i} k_{\sigma(r)} .
$$

Using the conventions and notation introduced in appendix A one arrives at the following Feynman representation of the tree-level amplitude (3.6) given in [52]

$A_{n ; q}^{\mathrm{tree}}\left(p_{a}^{+}, p_{b}^{-} ; k_{1}, \ldots, k_{n}\right)=\frac{(-e / \sqrt{2})^{n}}{\prod_{i=1}^{n}\left\langle p_{\text {ref }}^{i,-h_{i}} \mid k_{i}^{h_{i}}\right\rangle} \sum_{\sigma \in \mathfrak{S}_{n}}\left\langle a_{\sigma(1)} p_{a}\right\rangle\left[p_{b} b_{\sigma(n)}\right] \prod_{i=1}^{n-1} \frac{\left\langle a_{\sigma(i+1)}\left|q_{i}\right| b_{\sigma(i)}\right]}{q_{i}^{2}}$.

Here $\left\langle p^{-} \mid q^{+}\right\rangle=\langle p q\rangle$ and $\left\langle p^{+} \mid q^{-}\right\rangle=[p q]$. In the above expression we have made the helicity choice $h=\frac{1}{2}$. The other amplitude with $h=-\frac{1}{2}$ can be obtained by charge conjugation. The reference momentum $p_{\text {ref }}^{i}$ of the $i^{\text {th }}$ photon is an arbitrary light-like momentum. It cancels in the physical amplitude. We have employed the same notation as introduced in $[52]$

$$
a_{i}=\frac{1+h_{i}}{2} p_{\mathrm{ref}}^{i}+\frac{1-h_{i}}{2} k_{i}, \quad b_{i}=\frac{1+h_{i}}{2} k_{i}+\frac{1-h_{i}}{2} p_{\mathrm{ref}}^{i} .
$$

For a generic photon helicity configuration this sum would contain $n$ factorial terms. Choosing the reference momentum of the $h=+1$ helicity photons to be $p_{a}$ and the reference momentum of the $h=-1$ helicity photons to be $p_{b}$ so that

$$
p_{\text {ref }}^{i}=\frac{1+h_{i}}{2} p_{a}+\frac{1-h_{i}}{2} p_{b}
$$

one sees that the amplitude with $n_{+}$photons of helicity $h_{+}=+1$ and $n_{-}$photons of helicity $h_{-}=-1$ has only $n_{+} \times n_{-} \times\left(n_{+}+n_{-}-2\right)$ ! non-zero contributions in the sum (3.6) or (3.9).

With the choice of reference momentum eq. (3.11) one sees that, if all the helicities of the photons are $h=+1$ or all are of helicity $h=-1$ each term in the sum (3.6) vanish. This is in agreement with the supersymmetric Ward identities [53-56] for supersymmetric QED.

Choosing the specific MHV helicity configuration (with one negative helicity photon in the $1^{\text {st }}$ position and all the rest positive), the amplitude takes the form of the sum of permutations of ordered MHV Parke-Taylor [54] amplitudes

$$
A_{n ; q}^{\text {tree }}\left(p_{a}^{+}, p_{b}^{-} ; k_{1}^{-}, k_{2}^{+}, \ldots, k_{n}^{+}\right)=\frac{e^{n}}{2^{\frac{n}{2}}} \frac{\left\langle p_{a} k_{i}\right\rangle^{3}\left\langle p_{b} k_{i}\right\rangle}{\left\langle p_{a} p_{b}\right\rangle^{2}} \sum_{\sigma \in \mathfrak{S}_{n}} \frac{1}{\left\langle p_{a} k_{\sigma(1)}\right\rangle\left\langle k_{\sigma(1)} k_{\sigma(2)}\right\rangle \cdots\left\langle k_{\sigma(n)} p_{b}\right\rangle} .
$$

By making use of the eikonal identity given in [55]

$$
\sum_{\sigma \in \mathfrak{S}_{n}} \frac{\left\langle k_{a} k_{b}\right\rangle}{\left\langle k_{a} k_{\sigma(1)}\right\rangle\left\langle k_{\sigma(2)} k_{\sigma(3)}\right\rangle \cdots\left\langle k_{\sigma(n)} k_{b}\right\rangle}=\prod_{1 \leq r \leq n} \frac{\left\langle k_{a} k_{b}\right\rangle}{\left\langle k_{i} k_{r}\right\rangle\left\langle k_{j} k_{r}\right\rangle},
$$


one obtains the compact form

$$
A_{n ; q}^{\text {tree }}\left(p_{a}^{+}, p_{b}^{-} ; k_{1}^{+}, \ldots, k_{i-1}^{+}, k_{i}^{-}, k_{i+1}^{+}, \ldots, k_{n}^{+}\right)=\frac{e^{n}}{2^{\frac{n}{2}}} \frac{\left\langle p_{a} p_{b}\right\rangle^{n-2}\left\langle p_{a} k_{i}\right\rangle^{3}\left\langle p_{b} k_{i}\right\rangle}{\prod_{j=1}^{n}\left\langle p_{a} k_{j}\right\rangle\left\langle p_{b} k_{j}\right\rangle} .
$$

It was shown in ref. [57] that the $N^{k} \mathrm{MHV}$ amplitude can be constructed using a CSW construction [58] or via BCFW [59] recursion relations.

\subsubsection{Massive scalar tree amplitudes}

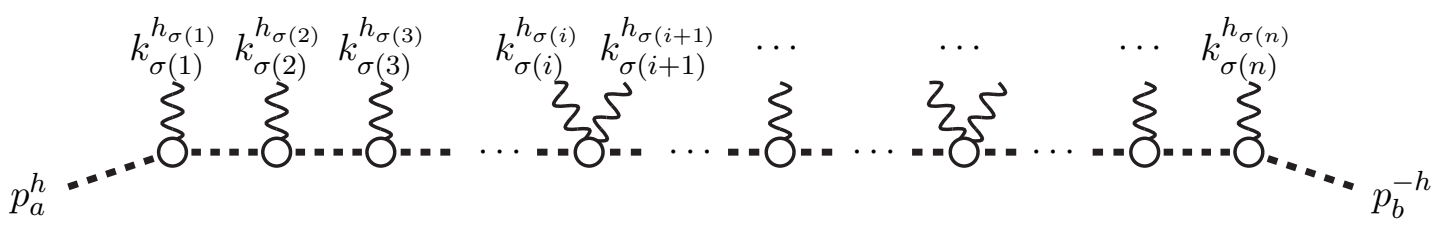

Figure 3: Tree-level scalar Feynman diagram.

In this section we examine the massive scalar amplitudes

$$
\varphi\left(p_{a}\right)+\varphi^{*}\left(p_{b}\right)+\gamma\left(k_{1}\right)+\cdots+\gamma\left(k_{n}\right) \rightarrow 0
$$

The tree-level amplitude with $n$ external photons attached to a scalar line is built from cubic and quartic vertices of the QED Lagrangian (again we will refer to ref. [51] for details on the Feynman rules). The amplitude is the sum over the permutations

$$
A_{n ; \varphi}^{\text {tree }}\left(p_{a}^{0}, p_{b}^{0} ; k_{1}, \ldots, k_{n}\right)=e^{n} \sum_{\sigma \in \mathfrak{S}_{n}} \mathcal{A}_{n ; \varphi}^{\text {tree }}\left(p_{a}^{0}, p_{b}^{0} ; k_{\sigma(1)}, \ldots, k_{\sigma(n)}\right),
$$

of an amplitude defined from the partition of the $n$ ordered external legs partitioned in group of at most length two

$$
\mathcal{A}_{n ; \varphi}^{\mathrm{tree}}\left(p_{a}^{0}, p_{b}^{0} ; k_{\sigma(1)}, \ldots, k_{\sigma(n)}\right)=\sum_{\substack{a_{1}+\cdots+a_{r}=n \\ a_{k} \in\{1,2\}}} \prod_{s=1}^{r} \frac{\epsilon_{\sigma\left(a_{1}+\cdots+a_{s-1}+1\right)} \cdot H\left(a_{s}\right)}{\left(p_{a}+\sum_{j=1}^{a_{1}+\cdots+a_{s}} k_{\sigma(j)}\right)^{2}-\mu^{2}},
$$

with

$$
H\left(a_{s}\right)= \begin{cases}q+\sum_{j=1}^{a_{1}+\cdots+a_{s-1}} k_{\sigma(j)} & \text { if } a_{s}=1 \\ \epsilon_{\sigma\left(a_{1}+\cdots+a_{s}\right)} & \text { if } a_{s}=2 .\end{cases}
$$

Because of the cubic and quartic vertices, this amplitude is a much larger sum of terms than the fermionic case, since the scalar $n$-photon tree-amplitude is a sum over $n ! \times F_{n+1}$ where $F_{r}$ is the Fibonacci number of order $r$ (such that $F_{0}=F_{1}=1$ and $\left.F_{2}=2\right){ }^{2}$

\footnotetext{
${ }^{2}$ The tree-level $A_{n ; \varphi}^{\text {tree }}$ photon scalar amplitude is constructed by adding one external photon line connected with a cubic vertex to the $n-1$ amplitude $A_{n-1 ; \varphi}^{\text {tree }}$ or by adding two photon lines connected by the quartic vertex to the $n-2$ amplitude $A_{n-2 ; \varphi}^{\text {tree }}$. Therefore the number of ordered amplitudes $F_{n}$ satisfies the Fibonacci recursion relation $F_{n}=F_{n-1}+F_{n-2}$.
} 


\subsection{BCFW shifts and large momentum scaling at tree-level}

In this section we will analyse the large $z$ scaling behaviour of photon tree-amplitudes under the BCFW shift [59]. This is done for cases of photons coupled to a massless fermion pair and a massive scalar pair which is useful for our investigation of which scalar integrals appear in the $n$-photon one-loop amplitude as described earlier in this section.

Cancellations in one-loop graviton scattering has already been studied through relating the coefficients of a scalar integral basis of the one-loop amplitude and the scaling behaviour of their corresponding unitarity cuts, i.e., products of on-shell tree amplitudes, under BCFW shifts $[6,7,25]$.

For example the large $z$ limit of the BCFW shift of the cut propagator momenta is related [7] to the large $t$ limit in the triple cut for triangles (see section C.1) using Forde's parametrisation of the cut loop momentum [43]. Similarly (see section C.2) the large $z$ scaling is related [7] to the large $y^{2} / t$ scaling in the double cut in Forde's parametrisation of the cut loop momentum. The large $z$ limit of the BCFW scaling behaviour for the cut tree amplitude can be used directly to test if the one-loop amplitude has any scalar triangle and bubble integrals.

Rational pieces in the amplitude can be probed for in a similar way using $D$-dimensional unitarity techniques $[41,42,45,60,61]$. One can then relate the large $z$ limit of the BCFW shift to the large momentum limit of a massive cut loop momentum following the methods of $[43,47]$.

\subsubsection{Large- $z$ scaling for the massless tree amplitudes}

Since the fermion line carries the loop momentum in the $n$-photon amplitude the relevant BCFW shift for the fermion tree amplitudes is that of shifting the quark, $p_{a}$, and anti-quark, $p_{b}$ :

$$
\begin{aligned}
& \text { type } \left.\left.\left.s=+1, \quad\left|\widehat{p}_{a}\right\rangle \equiv\left|p_{a}\right\rangle+z\left|p_{b}\right\rangle, \quad \mid \widehat{p}_{b}\right] \equiv \mid p_{b}\right]-z \mid p_{a}\right], \\
& \text { type } \left.\left.\left.s=-1, \quad\left|\widehat{p}_{b}\right\rangle \equiv\left|p_{b}\right\rangle+z\left|p_{a}\right\rangle, \quad \mid \widehat{p}_{a}\right] \equiv \mid p_{a}\right]-z \mid p_{b}\right] .
\end{aligned}
$$

Under these shifts the polarisation of the external fermions behave as

$$
u_{ \pm}\left(\widehat{p}_{a}\right)=u_{ \pm}\left(p_{a}\right)+z \frac{s \pm 1}{2} u_{ \pm}\left(p_{b}\right), \quad u_{ \pm}\left(\widehat{p}_{b}\right)=u_{ \pm}\left(p_{b}\right)+z \frac{s \mp 1}{2} u_{ \pm}\left(p_{a}\right),
$$

while the propagator factors $\not q_{i}=K_{\sigma(i)}+\not p_{a}$ shift according to

$$
\widehat{q}_{i}=q_{i}+z \pitchfork,
$$

where $\pi$ is a light-like vector defined by

$$
丸 \equiv \frac{1+s}{2}\left(\left|p_{b}\right\rangle\left[p_{a}|+| p_{a}\right]\left\langle p_{b}\right|\right)+\frac{s-1}{2}\left(\left|p_{a}\right\rangle\left[p_{b}|+| p_{b}\right]\left\langle p_{a}\right|\right) .
$$

The fermion tree amplitudes in eq. (3.9) and the scalar tree amplitude of eq. (3.17) have the following behaviour in the large $z$ limit

$$
\lim _{z \rightarrow \infty} A_{n ; q}^{\text {tree }}\left(p_{a}^{h}, p_{b}^{-h} ; k_{1}, \ldots, k_{n}\right) \sim C_{q}^{\infty}\left(p_{a}^{h}, p_{b}^{-h} ; k_{1}, \ldots, k_{n}\right) \times \frac{z^{2 h s}}{z^{n-2}},
$$


where $s=+1$ for the shift (3.19) and $s=-1$ for the shift 3.20 ) and $h= \pm \frac{1}{2}$ for a fermion and $h=0$ for a scalar.

We have checked this behaviour numerically for all helicity configurations up to $n=10$ photon lines. For the case of the fermionic tree amplitude a formal proof of this behaviour is given in the appendix $\mathrm{B}$. The case for the massless scalar amplitude follows directly from the fermionic case by using the $\mathcal{N}=1$ super-QED Ward identities.

\subsubsection{Large- $z$ scaling of the massive scalar tree amplitudes}

To analyse the rational contributions to the one-loop photon amplitudes we must consider a BCFW shift of the two massive scalar particles in the tree amplitude given by eq. (3.17).

In order to solve the on-shell conditions for a recursive construction of an amplitude while shifting two massive particles it is necessary to define two additional massless vectors [62]. We therefore define a pair of "flattened" vectors from a pair a massive vectors $p_{a}, p_{b}$ each with mass $\mu$ :

$$
p_{a}=p_{a}^{b}+\frac{\mu^{2}}{\gamma} p_{b}^{b} ; \quad p_{b}=p_{b}^{b}+\frac{\mu^{2}}{\gamma} p_{a}^{b},
$$

where $\left(p_{a}^{b}\right)^{2}=0$ and $\left(p_{b}^{b}\right)^{2}=0$ and

$$
\gamma=2\left(p_{a}^{b} \cdot p_{b}^{b}\right)=\left(p_{a} \cdot p_{b}\right)\left(1+\sqrt{1-\frac{\mu^{4}}{\left(p_{a} \cdot p_{b}\right)^{2}}}\right),
$$

so that $p_{a}^{b} \rightarrow p_{a}$ and $p_{b}^{b} \rightarrow p_{b}$ for $\mu^{2} \rightarrow 0$. For fixed $\mu^{2}$ we define the shift as

$$
\left.\left.\left.\left|\widehat{p}_{a}^{b}\right\rangle=\left|p_{a}^{b}\right\rangle+z\left|p_{b}^{b}\right\rangle ; \quad \mid \widehat{p}_{b}^{b}\right]=\mid p_{b}^{b}\right]-z \mid p_{a}^{b}\right],
$$

implying that the original momenta are shifted according to

$$
\widehat{p}_{a}=\not p_{a}+z\left(1-\frac{\mu^{2}}{\gamma}\right)\left|p_{b}^{b}\right\rangle\left[p_{a}^{b}\left|; \quad \widehat{p}_{b}=\not p_{b}-z\left(1-\frac{\mu^{2}}{\gamma}\right)\right| p_{a}^{b}\right\rangle\left[p_{b}^{b} \mid .\right.
$$

In this case the large $z$ limit of the shifted propagators in (3.17) become

$$
\lim _{z \rightarrow \infty} \frac{1}{\left(\widehat{p}_{a}+K\right)^{2}-\mu^{2}} \sim \frac{1}{z(\pi \cdot K)} \frac{1}{1-\mu^{2} / \gamma}
$$

where $\pi$ is defined as in eq. (3.22) with $p_{a}$ and $p_{b}$ replaced by $p_{a}^{b}$ and $p_{b}^{b}$ respectively. We have in this argument used that $\pi \cdot p_{a}=0$. At the leading order in $\mu^{2}$ the asymptotic value of the propagators take a similar form to the one appearing in the massless case. This indicates that the leading large $z$ behaviour of the massive scalar tree amplitudes is the same as in the massless case

$$
\lim _{z \rightarrow \infty} A_{n ; \varphi}^{\text {tree }}\left(p_{a}^{h}, p_{b}^{-h} ; k_{1}, \ldots, k_{n}\right)=\frac{1}{z^{n-2}} C_{\varphi}^{\infty}\left(p_{a}^{h}, p_{b}^{-h} ; k_{1}, \ldots, k_{n} \mid \mu^{2}\right) .
$$

We have checked this behaviour numerically up to $n=8$ external photons and for all helicity configurations. 
The coefficient of $C_{\varphi}^{\infty}$ depends on the mass $\mu^{2}$, and in the massless limit $\mu^{2} \rightarrow 0$ the behaviour of a massless scalar tree amplitude is recovered. In the particular cases of the all-plus or all-minus photon helicity configuration, which vanish in the massless limit, the contribution $C_{\varphi}^{\infty}\left(p_{a}^{h}, p_{b}^{-h} ; k_{1}^{+}, \ldots, k_{n}^{+} \mid \mu^{2}\right)=O\left(\mu^{2}\right)$. It can be seen that these sub-leading contributions play an important role in the analysis of the potential rational pieces in appendix C.3.

For large $\mu$ we express the massive momenta in terms of massless momenta using

$$
p_{a}=\mu p_{a}^{b}+\frac{\mu}{\gamma} p_{b}^{b} ; \quad p_{b}=\frac{1}{\mu} p_{b}^{b}+\frac{\mu^{3}}{\gamma} p_{a}^{b},
$$

with the same definition for $\gamma$. To write this solution we used the freedom to rescale the massless momenta $\left(p_{a}^{b}, p_{b}^{b}\right) \rightarrow\left(\lambda p_{a}^{b}, \lambda^{-1} p_{b}^{b}\right)$. We have chosen a linear scaling in $\mu$ since this is what will be needed in the appendix C.3 for analysing the eventual rational pieces from boxes.

For large $\mu$ we have that

$$
\lim _{\mu \rightarrow \infty} \frac{\gamma}{\mu^{2}}=i \operatorname{sign}\left(p_{a} \cdot p_{b}\right)
$$

and in this limit

$$
p_{a} \sim \mu p_{a}^{b} ; \quad p_{b} \sim-i \mu \frac{p_{a}}{\operatorname{sign}\left(p_{a} \cdot p_{b}\right)} .
$$

In this case the asymptotic form of the propagators is given by

$$
\lim _{\mu^{2} \rightarrow \infty} \frac{1}{\left(p_{a}+K\right)^{2}-\mu^{2}} \sim \frac{1}{2 \mu\left(p_{a}^{b} \cdot K\right)},
$$

which is of the same form as for the BCFW shift of the massless amplitude with $z \sim \mu$. In this case the tree amplitudes have the asymptotic behaviour given in eq. (3.29) with $z=\mu$.

These results are used in the appendix C.3 where the rational piece contributions to the one-loop amplitude are analysed.

\subsubsection{Origin of the improved BCFW scaling behaviour}

In this section we examine the analytic structure of the cancellations that give rise to the improved BCFW scaling behaviour observed in the preceding sections. We make use of a specific gauge choice which makes the cancellations manifest in each of the contributing diagrams. However, we will see that this is not sufficient in order to remove the role of the summation of external orderings for anything but the simplest Abelian amplitudes.

The analysis in the previous sections (and the appendix B) showed that the treeamplitudes in QED are extremely well behaved in the large $z$ limit of the BCFW shifts

$$
\lim _{z \rightarrow \infty} \sum_{\sigma \in \mathfrak{S}_{n}} \mathcal{A}_{n}(z) \sim \frac{z^{2 h s}}{z^{n-2}} .
$$

For the case of the massless fermion tree-amplitudes this property can be proven diagram by diagram by using a special gauge choice. However this technique is not sufficient to 
find the observed scaling property in more complicated amplitudes containing higher point interactions such as the massive scalar tree amplitude and those in gravity. In the discussion below we give explicit examples of how the sum over external orderings is responsible for the remaining cancellations.

In a Feynman graph based analysis of the scalar tree-amplitudes one can observe that the cubic vertex at worst scales like $O(z)$, the quartic vertex like $O(1)$ and that the ordered amplitudes in eq. (3.17) scale at worst like

$$
\lim _{z \rightarrow \infty} \mathcal{A}_{n ; \varphi}^{\text {tree }}(z) \sim z
$$

By taking the transverse gauge $\pi \cdot \epsilon^{h_{i}}=0$ for all the photons by setting the reference momenta to be $p_{\text {ref }}=\pi$ where $\pi$ is the light-like momentum defined in eq. (3.22), then all cubic vertices are independent of $z$ and scale as $O(1)$. In this gauge the ordered amplitudes have the large $z$ scaling $\mathcal{A}_{n}(z) \sim 1 / z^{n / 2-1}$ dominated by graphs with the maximum number of quartic vertices. The sum over the orderings of external legs improves this behaviour to the optimal one (3.34) as can be seen from the following four-photon and five-photon examples. With the choice of gauge $p_{\text {ref }}=\pi$ only the contractions between polarisations of opposite helicities are non-vanishing

$$
\epsilon_{i}^{ \pm}\left(\pi, k_{i}\right) \cdot \epsilon_{j}^{ \pm}\left(\pi, k_{j}\right)=0 ; \quad \epsilon_{i}^{ \pm}\left(\pi, k_{i}\right) \cdot \epsilon_{j}^{\mp}\left(\pi, k_{j}\right) \neq 0
$$

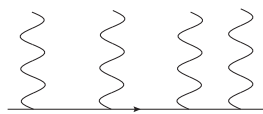

(i)

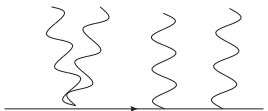

(ii)

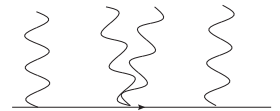

(ii)

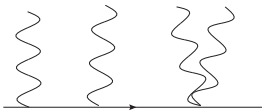

(ii)

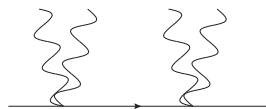

(iii)

Figure 4: The $e^{-} e^{+} \rightarrow 4 \gamma$ tree-amplitude is composed by the Feynman diagrams built from (i) four three-point vertices, (ii) two three-point vertices and one four-point vertex, and (iii) two four-point vertices. The label of the external photon have to be symmetrically distributed over the photon lines.

We will be considering as a first example the four-photon amplitude with the helicity configuration $(--++)$. For a colourless theory the orderings of the external legs does not matter. The tree-amplitude is built from permutations of the four topologies shown in figure 4 : (i) four three-point vertices, which have the large $z$ scaling $O\left(1 / z^{3}\right)$, (ii) two three-point vertices and one four-point vertex, which scales as $O\left(1 / z^{2}\right)$, (iii) two four-point vertices, which has the scaling $O(1 / z)$.

Each ordered contribution with two four-point vertices has a large $z$ behaviour that is worse than the observed behaviour for the total amplitude. We show that this bad scaling is cancelled in the sum over orderings of external legs.

The contribution from all the four-point vertices to the four-photon amplitude is given by 


$$
\begin{aligned}
\sum_{\sigma_{2} \times \sigma_{2}} & \frac{\varepsilon_{\sigma(1)}^{h_{\sigma(1)}} \cdot \varepsilon_{\sigma(2)}^{h_{\sigma(2)}} \varepsilon_{\sigma(3)}^{h_{\sigma(3)}} \cdot \varepsilon_{\sigma(4)}^{h_{\sigma(4)}}}{\left(\widehat{p}_{a}+K_{\sigma(1) \sigma(2)}\right)^{2}-\mu^{2}} \\
& =\frac{\varepsilon_{1}^{-} \cdot \varepsilon_{3}^{+} \varepsilon_{2}^{-} \cdot \varepsilon_{4}^{+}}{z \alpha \pi \cdot K_{13}}+\frac{\varepsilon_{2}^{-} \cdot \varepsilon_{4}^{+} \varepsilon_{1}^{-} \cdot \varepsilon_{3}^{+}}{z \alpha \pi \cdot K_{24}}+\frac{\varepsilon_{1}^{-} \cdot \varepsilon_{4}^{+} \varepsilon_{2}^{-} \cdot \varepsilon_{3}^{+}}{z \alpha \pi \cdot K_{14}}+\frac{\varepsilon_{2}^{-} \cdot \varepsilon_{3}^{+} \varepsilon_{1}^{-} \cdot \varepsilon_{4}^{+}}{z \alpha \pi \cdot K_{23}}+\mathcal{O}\left(z^{-2}\right) \\
& =\varepsilon_{1}^{-} \cdot \varepsilon_{3}^{+} \frac{\pi \cdot K_{1234}}{z^{2} \alpha^{2} \pi \cdot K_{13} \pi \cdot K_{24}}+\varepsilon_{1}^{-} \cdot \varepsilon_{4}^{+} \frac{\pi \cdot K_{1234}}{z^{2} \alpha^{2} \pi \cdot K_{14} \pi \cdot K_{23}}+\mathcal{O}\left(z^{-2}\right) \\
& =\mathcal{O}\left(z^{-2}\right),
\end{aligned}
$$

where $\sigma_{2} \times \sigma_{2}$ means that one has to sum over the 2-cycle decompositions of the permutations. The cancellation arises because of momentum conservation $k_{1}+\cdots+k_{4}=-p_{a}-p_{b}$ and via $\pi \cdot p_{a}=0$ and $\pi \cdot p_{b}=0$.

Using a more compact notation we have showed that

$$
\left(1^{-}, 3^{+}\right) \cdot\left(2^{-}, 4^{+}\right)+\left(2^{-}, 4^{+}\right) \cdot\left(1^{-}, 3^{+}\right) \underset{z \rightarrow \infty}{\rightarrow} \mathcal{O}\left(z^{-2}\right)
$$

In the case of the five-photon amplitude with the helicity configuration $(--+++)$, we find that the cancellations now involves all twelve different orderings:

$$
\begin{aligned}
& \left(3^{+}\right) \cdot\left(1^{-}, 4^{+}\right) \cdot\left(2^{-}, 5^{+}\right)+\left(3^{+}\right) \cdot\left(2^{-}, 5^{+}\right) \cdot\left(1^{-}, 4^{+}\right) \\
+ & \left(1^{-}, 4^{+}\right) \cdot\left(3^{+}\right) \cdot\left(2^{-}, 5^{+}\right)+\left(2^{-}, 5^{+}\right) \cdot\left(3^{+}\right) \cdot\left(1^{-}, 4^{+}\right) \\
+ & \left(1^{-}, 4^{+}\right) \cdot\left(2^{-}, 5^{+}\right) \cdot\left(3^{+}\right)+\left(2^{-}, 5^{+}\right) \cdot\left(1^{-}, 4^{+}\right) \cdot\left(3^{+}\right)+(4 \leftrightarrow 5) \\
\propto & \frac{\varepsilon_{3}^{+} \cdot\left(2 p_{a}+K_{1245}\right)}{z^{2}}\left(\frac{1}{\pi \cdot K_{14} \pi \cdot K_{25}}+\frac{1}{\pi \cdot K_{3} \pi \cdot K_{14}}+\frac{1}{\pi \cdot K_{3} \pi \cdot K_{25}}\right)+(4 \leftrightarrow 5) \\
= & \frac{\varepsilon_{3}^{+} \cdot\left(2 p_{a}+K_{1245}\right)}{z^{2}} \frac{\pi \cdot K_{12345}}{\pi \cdot K_{14} \pi \cdot K_{3} \pi \cdot K_{25}}+(4 \leftrightarrow 5) \\
= & \mathcal{O}\left(z^{-3}\right) .
\end{aligned}
$$

We have used momentum conservation $k_{1}+\cdots+k_{5}=-p_{a}-p_{b}$ and that $\pi \cdot p_{a}=0$ and $\pi \cdot p_{b}=0$. The first step requires that the terms from the $(4 \leftrightarrow 5)$ exchange but the relation is independent of the momentum appearing in the single three-point interaction. It is therefore sufficient to show that the five-photon amplitudes scale as $1 / z^{3}$ as required.

In the gravity case we have a similar phenomenon. The multi-graviton vertices naïvely scale as $z^{2}$ in the large $z$ limit and the ordered Feynman graphs scale at worst like $z^{n-1} \times$ $z^{f\left(h_{1}, h_{2}, s\right)}$. Here $f\left(h_{1}, h_{2}, s\right) \in[-4,4]$ is an integer valued function of the polarisations $h_{1,2}$ of the shifted legs and the type of shift $s= \pm 1$. By considering the transverse gauge

$$
\pi^{\mu} \epsilon_{\mu \nu}^{h_{i}}\left(\pi, k_{i}\right)=0 ; \quad \epsilon_{\mu \nu}^{h_{i}}\left(\pi, k_{i}\right) \pi^{\nu}=0
$$

and by setting the reference momentum of the unshifted legs to be $p_{\text {ref }}=\pi$, the multigraviton vertices that do not involve the two shifted legs scale at most as $O(z)$ and the worst scaling of the ordered Feynman graphs is thus given by the $z$ dependence of the polarisation of the shifted legs $z^{2} z^{f\left(h_{1}, h_{2}, s\right)}$. The sum over the orderings of the external legs improves the scaling behaviour of the total amplitude to either $z^{-2}$ or $z^{6}$ depending on the polarisation of the shifted states $[5,6,63,64]$. 


\subsection{One-loop structure from large $z$ momentum scaling}

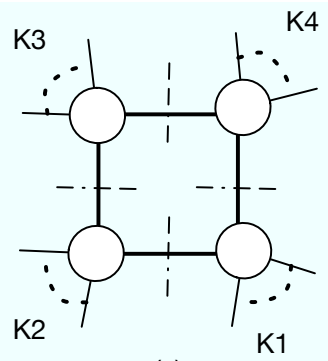

(a)

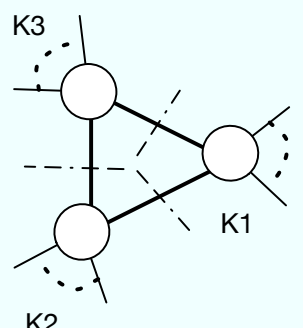

(b)

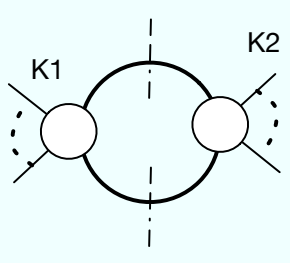

(c)

Figure 5: Representation of (a) the quadruple cut contributing to the coefficient $c_{4 ; K_{1}\left|K_{2}\right| K_{3} \mid K_{4}}$, (b) the triple cut contributing to the coefficient $c_{4 ; K_{1}\left|K_{2}\right| K_{3}}$ and (c) the double cut contributing to the coefficient $c_{2 ; K_{1} \mid K_{2}}$ of the one-loop amplitude.

The generic decomposition of a $n$-photon one-loop amplitude in dimensional regularisation with four-dimensional external momenta is given by

$$
\begin{aligned}
A_{n ; q}^{\text {one-loop }} & =\sum_{\left\{K_{i}\right\}} c_{4 ; K_{1}\left|K_{2}\right| K_{3} \mid K_{4}} I_{4}^{\left(K_{1}\left|K_{2}\right| K_{3} \mid K_{4}\right)}+\sum_{\left\{K_{i}\right\}} c_{3 ; K_{1}\left|K_{2}\right| K_{3}} I_{3}^{\left(K_{1}\left|K_{2}\right| K_{3}\right)} \\
& +\sum_{\left\{K_{i}\right\}} c_{2 ; K_{1} \mid K_{2}} I_{2}^{\left(K_{1} \mid K_{2}\right)}+R_{n}+\mathcal{O}(\epsilon) .
\end{aligned}
$$

Here $I_{4}^{\left(K_{1}\left|K_{2}\right| K_{3} \mid K_{4}\right)}, I_{3}^{\left(K_{1}\left|K_{2}\right| K_{3}\right)}, I_{2}^{\left(K_{1} \mid K_{2}\right)}$ and $R_{n}$ are scalar box, triangle, bubble integrals and rational terms respectively evaluated in $D=4-2 \epsilon$ dimensions $[8,65]$. The coefficients $c_{4 ; K_{1}\left|K_{2}\right| K_{3} \mid K_{4}}^{i}, c_{3 ; K_{1}\left|K_{2}\right| K_{3}}^{j}$ and $c_{2 ; K_{1} \mid K_{2}}^{k}$ are associated with the corresponding scalar integral functions represented in fig. 5 where $K_{i}$ are the sum of the momenta at each vertex of the scalar integral functions.

In this section we will outline the consequences of the large momentum scaling at treelevel for the structure of the one-loop amplitude. The details of our analysis can be found in appendix $\mathrm{Q}$.

\subsubsection{Vanishing of triangle coefficients}

Parametrising the unfixed integration of the triple cut by a complex parameter $t$ leaves the triangle coefficients completely determined [43]. In the appendix C.1 we relate the large $t$-scaling of the tree amplitudes in the cut to the large $z$ behaviour under the BCFW shift (see as well ref. [7]). We show in the appendix that the large $t$ scaling is independent of the helicity of the state running in the loop $(h= \pm 1 / 2$ for the fermion or $h=0$ for the scalar)

$$
A_{n}^{\text {tree }} \underset{t \rightarrow \infty}{\rightarrow} \frac{C_{q}^{\infty}\left(K_{1}, K_{2}\right)}{t^{n-2}} .
$$

We will show in the appendix C.1 that the scaling behaviour of the tree amplitude in the cut leads us directly to the result expected from the world-line analysis: namely that all triangle coefficients vanish for one-loop $n>6$ amplitudes. This property is unexpected from naïve power counting and from the conjecture for the NNMHV amplitudes of ref. [37]. 


\subsubsection{Vanishing of bubble coefficients}

As described in appendix C.2 the above scaling behaviour gives sufficient information for concluding: that all bubble coefficients vanish for $n \geq 6$. For the case of $n=5$ the coefficients vanish after the sum over the orderings of the external legs is performed. This is in agreement with Furry's theorem. The result that no scalar bubble integral functions are present in multi-photon one-loop amplitude with $n \geq 6$ legs is in complete agreement with the world-line formalism of section 2. Our results although unexpected from naïve power counting also fits the analysis of the MHV amplitude given in [66] and the result for the six-point NMHV amplitudes computed in [36,37].

\subsubsection{Vanishing of rational terms}

In section C.3 we show that the $n$-photon amplitude with $n \geq 5$ cannot have rational polynomial contributions. The result of this analysis confirms the world-line string based result that there are no rational contributions for photon amplitudes with $n>4$. From a field theory perspective, the rational polynomial contributions can be ruled out via an analysis of the massive scalar tree amplitudes in the large mass limit as described in ref. [47].

This is in perfect agreement with the direct computation of Mahlon [38] of the finite helicity configurations:

$$
\begin{aligned}
A_{4 ; q}^{\text {one-loop }}\left(k_{1}^{+}, k_{2}^{+}, k_{3}^{+}, k_{4}^{+}\right) & =e^{4} \frac{\left[k_{1} k_{2}\right]\left[k_{3} k_{4}\right]}{\left\langle k_{1} k_{2}\right\rangle\left\langle k_{3} k_{4}\right\rangle}, \\
A_{4 ; q}^{\text {one-loop }}\left(k_{1}^{+}, k_{2}^{+}, k_{3}^{+}, k_{4}^{-}\right) & =e^{4} \frac{\left[k_{1} k_{2}\right]\left[k_{2} k_{3}\right]\left\langle k_{3} k_{1}\right\rangle}{\left\langle k_{1} k_{2}\right\rangle\left\langle k_{2} k_{3}\right\rangle\left[k_{3} k_{1}\right]}, \\
A_{n ; q}^{\text {one-loop }}\left(k_{1}^{+}, \ldots, k_{n}^{+}\right)=0 & \text { for } n \geq 5, \\
A_{n ; q}^{\text {one-loop }}\left(k_{1}^{+}, \ldots, k_{n-1}^{+}, k_{n}^{-}\right)=0 & \text { for } n \geq 5 .
\end{aligned}
$$

It is also in agreement with the $n$-point MHV computation of Bernicot et al. [37] and the known six-point computations of refs. [36,37].

\subsection{No-triangle property of one-loop photon amplitudes}

The vanishing of the coefficients described above leads us to the expected result previously obtained via the world-line analysis given by eq. (2.23). The multi-photon fermionic or scalar one-loop amplitudes with $n \geq 8$ external photons satisfy a no triangle property, and hence contain solely scalar box integral functions in $D=4-2 \epsilon$ dimensions. The $n$ point amplitude can thus be written as

$$
A_{n ; \Phi}^{\text {one-loop }}=\sum_{i} c_{4}^{i} I_{4}^{(i)}
$$

where $\Phi=q$ for the fermionic loop or $\Phi=\varphi$ for the complex scalar loop.

The above result is expressed in terms of scalar box integral functions evaluated in $D=4-2 \epsilon$ that carry $\epsilon$ singularities. Because of manifest one-loop ultra-violet and infra-

red finiteness of the one-loop amplitude both the $1 / \epsilon^{2}$ and $1 / \epsilon$ poles must cancel between the various terms in the amplitude. Because the amplitude reduces to scalar box contributions 
it is immediate (but tedious) to evaluate the expression for the amplitude from its quadruple cut. It would very be interesting to understand this generic structure valid for all helicity configurations from a dual Wilson loop description as in $[67,68]$. This in turn implies relations between the box coefficients which will be discussed in the subsequent section.

\subsection{Universal ultra-violet and infra-red pole structure}

The infra-red singularities in an ordered gauge theory one-loop amplitude is described by $[69]$

$$
\left.\mathcal{A}_{n}^{\text {one-loop }}\left(k_{1}, \ldots, k_{n}\right)\right|_{I R} \propto \sum_{i=1}^{n} \frac{\left(-\left(k_{i}+k_{i+1}\right)^{2}\right)^{-\epsilon}}{\epsilon^{2}} \mathcal{A}_{n}^{\text {tree }}\left(k_{1}, \ldots, k_{n}\right) .
$$

Since the $n$-photon tree-level amplitude are vanishing in an Abelian theory like QED, there is no infra-red singularities in the $n$-photon amplitudes at one-loop. As a result, previous calculations $[36-38,66,70,71]$ were expressed in terms of the finite part $\mathcal{F}_{4}$ of the scalar box integral function $I_{4}$. This is because the dimensionless one-mass and two-mass triangle integral functions are given by (see the appendix $\mathrm{D}$ )

$$
\begin{aligned}
& \tilde{I}_{3}\left(k_{1}, k_{2}, K_{3}\right) \equiv\left(-K_{3}^{2}\right) I_{3}\left(k_{1}, k_{2}, K_{3}\right)=\frac{r_{\Gamma} \mu^{2 \epsilon}}{\epsilon^{2}}\left(-K_{3}^{2}\right)^{\epsilon}, \\
& \tilde{I}_{3}\left(k_{1}, K_{2}, K_{3}\right) \equiv\left(K_{2}^{2}-K_{3}^{2}\right) I_{3}\left(k_{1}, K_{2}, K_{3}\right)=\frac{r_{\Gamma} \mu^{2 \epsilon}}{\epsilon^{2}}\left(\left(-K_{2}^{2}\right)^{-\epsilon}-\left(-K_{3}^{2}\right)^{-\epsilon}\right),
\end{aligned}
$$

and we can define the finite parts $\mathcal{F}_{4}$ of the scalar box integral functions $I_{4}$ by subtracting dimensionless one-mass and two-mass divergent scalar triangle functions

$$
\mathcal{F}_{4}=I_{4}+\sum_{i} t_{i}^{1} \tilde{I}_{3}^{(i) 1-\text { mass }}+\sum_{i} t_{i}^{2} \tilde{I}_{3}^{(i) 2-\text { mass }} .
$$

Here $t_{i}^{1}$ and $t_{i}^{2}$ are some coefficients depending on the kinematic invariants which are given in the appendix $\mathrm{D}$. Choosing this basis makes the amplitude explicitly free from divergences but hides the no-triangle property given by equation (3.44). Because the dimensionless one-mass triangle gives the multi-particle infra-red divergence $\left(-K^{2}\right)^{-\epsilon} / \epsilon^{2}$, the absence of triangles lead to a set of relations between the box coefficients appearing in the decomposition (3.44). In the next section we will give for the specific example of MHV multi-photon amplitudes the relations between the box coefficients for the cancellation of the infra-red divergences.

We would like to contrast this to the gravity case where the infra-red singularities are given by [72]

$$
\left.\mathcal{M}_{n}^{\text {one-loop }}\left(k_{1}, \ldots, k_{n}\right)\right|_{I R} \propto \mathcal{M}_{n}^{\text {tree }}\left(k_{1}, \ldots, k_{n}\right) \sum_{i=1}^{n} \frac{\left(-\left(k_{i}+k_{i+1}\right)^{2}\right)^{1-\epsilon}}{\epsilon^{2}} .
$$

The leading $1 / \epsilon^{2}$ pole in the gravity amplitude cancels [73] because of the on-shell condition $\sum_{i=1}^{n}\left(k_{i}+k_{i+1}\right)^{2}=0$ but the amplitude has still a non-vanishing $1 / \epsilon$ pole contribution in $D=4-2 \epsilon$. 
By power counting, ultra-violet divergences can only occur in the three-photon and four-photon one-loop amplitudes. The three-photon amplitude vanishes by Furry's theorem while the four-photon amplitudes are non-vanishing. The four-photon amplitude at one-loop is dimensionless in four dimensions, and could have a logarithmic ultra-violet divergence. However such an ultra-violet divergence has to be associated with a local gauge invariant operator $T_{m_{1} n_{1} \cdots m_{4} n_{4}} F_{m_{1} n_{1}}^{1} \cdots F_{m_{4} n_{4}}^{4}$, for which the four photons amplitude is given by some combination of the four field-strengths $F_{m n}=\epsilon_{m} k_{n}-\epsilon_{n} k_{m}$ of the external photons. Because such an operator has mass dimension four, no ultra-violet divergences can occur by dimensional analysis. Therefore all multi-photon one-loop amplitudes are ultra-violet finite and all possible rational pieces contributions are of infra-red origin. The presence of rational contributions will be analysed in section C.3 following the method of $[43,47]$.

\subsubsection{The $n$-photon MHV Amplitude}

In this section we re-evaluate the $n$-photon one-loop MHV amplitude $A_{n ; q}^{\text {one-loop }}\left(k_{1}^{-}, k_{2}^{-}\right.$, $k_{3}^{+}, \ldots, k_{n}^{+}$) for $n \geq 8$. This amplitude was first computed by Mahlon in [66] and has been recently re-analysed using double unitarity cuts [37]. We present it again here in order to analyse the infra-red structure of the $n \geq 8$-photon MHV amplitudes which have only box contributions.

Because of the restrictions on the cut momenta [40] these MHV amplitudes are only given by the linear combination of the one-mass box $I_{4}^{1 m}\left(k_{i}^{+}, k_{a}^{-}, k_{j}^{+}, k_{b}^{-}+K_{1}^{+}\right)$with the massless legs given by the configurations $\left(k_{i}^{+}, k_{a}^{-}, k_{j}^{+}\right)$with $3 \leq i<j \leq n$ and $a, b=1,2$, and the massive leg $K_{1}^{+}=k_{3}+\cdots+k_{n}-k_{i}-k_{j}$. The two-mass easy box $I_{4}^{2 m e}\left(k_{i}^{+}, k_{a}^{+}+\right.$ $\left.K_{2}^{+}, k_{j}^{+}, k_{b}^{-}+K_{3}^{+}\right)$with the opposite massless legs is given by the configuration $\left(k_{i}^{+}, k_{j}^{+}\right)$ with $3 \leq i<j \leq n$ and the two massive legs $k_{a}^{-}+K_{2}^{+}$and $k_{b}^{-}+K_{3}^{+}$with $a, b=1,2$ and $K_{2}^{+}+K_{3}^{+}=k_{3}+\cdots+k_{n}-k_{i}-k_{j}$. Because the cut amplitude only involves MHV tree-amplitude factors we can make use of the compact formula of eq. (3.14) for the tree amplitudes in the cut leading to

$$
c_{4 ; k_{i}\left|k_{1}+K_{1}\right| k_{j} \mid k_{2}+K_{2}}=\left\langle k_{i}\left|K_{1}^{b}\right| k_{i}\right]\left\langle k_{j}\left|K_{1}^{b}\right| k_{j}\right] \times \tilde{c}_{4 ; k_{i}\left|k_{1}\right| k_{j} \mid k_{2}},
$$

with

$$
K_{1}^{b}=K_{1}-\frac{K_{1}^{2}}{\left\langle k_{1}\left|K_{1}\right| k_{i}\right]} k_{i}
$$

and

$$
\tilde{c}_{4 ; k_{i}\left|k_{1}\right| k_{j} \mid k_{2}}=\frac{1}{2}\left(\frac{\left\langle k_{1} k_{i}\right\rangle^{3}\left\langle k_{1} k_{j}\right\rangle^{2}\left\langle k_{2} k_{i}\right\rangle^{2}\left\langle k_{2} k_{j}\right\rangle^{3}}{\left\langle k_{i} k_{j}\right\rangle^{8}}+(1 \leftrightarrow 2)\right) \prod_{\substack{1 \leq r \leq n \\ r \neq i, j}} \frac{\left\langle k_{i} k_{j}\right\rangle}{\left\langle k_{i} k_{r}\right\rangle\left\langle k_{r} k_{j}\right\rangle} .
$$

Here $K_{1}+K_{2}$ is a repartition of the positive helicity states on each of the opposite corners of the box. The coefficient $\tilde{c}_{4 ; k_{i}\left|k_{1}\right| k_{j} \mid k_{2}}$ does not depend on the distribution of the positive helicity states of the opposite massive legs and gives the same expression for the one-mass box and the two-mass easy box. This is compatible with the soft limit relation between the two-mass easy box and the one-mass box

$$
\lim _{K_{2} \rightarrow 0} I_{4}^{2 m e}\left(k_{i}, k_{a}+K_{1}, k_{j}, k_{b}+K_{2}\right)=I_{4}^{1 m}\left(k_{i}, k_{a}+K_{1}, k_{j}, k_{b}\right) .
$$


Under the exchanges of the two positive helicity massless legs the two-mass-easy box coefficient has the parity

$$
c_{4, k_{i}\left|k_{1}\right| k_{j} \mid k_{2}}=(-1)^{n} c_{4, k_{j}\left|k_{1}\right| k_{i} \mid k_{2}} .
$$

This implies that the only amplitude with an even number of external photons lines is non-vanishing. This is a particular example of the consequence of Furry's theorem on the coefficients of the scalar box integrals.

Using that

$$
\left\langle k_{i}\left|K_{1}^{b}\right| k_{i}\right]\left\langle k_{j}\left|K_{1}^{b}\right| k_{j}\right]=\left(k_{i}+k_{1}+K_{1}\right)^{2}\left(k_{j}+k_{1}+K_{1}\right)^{2}-\left(k_{1}+K_{1}\right)^{2}\left(k_{2}+K_{2}\right)^{2},
$$

one can express the one-loop amplitude in terms of the dimensionless boxes $\tilde{I}_{4}^{2 m e}\left(k_{1}, K_{2}, k_{3}\right.$, $\left.K_{4}\right)=\left(s_{12} s_{23}-K_{2}^{2} K_{4}^{2}\right) I_{4}^{2 m e}\left(k_{1}, K_{2}, k_{3}, K_{4}\right)$

$$
A_{2 n ; q}^{\text {one-loop }}\left(k_{1}^{-}, k_{2}^{-}, k_{3}^{+}, \cdots, k_{2 n}^{+}\right)=\sum_{3 \leq i \neq j \leq 2 n} \tilde{c}_{4 ; k_{i}\left|k_{1}\right| k_{j} \mid k_{2}} \sum_{K_{1}^{(i j)}} \tilde{I}_{4}^{2 m e}\left(k_{i}, k_{1}+K_{1}^{(i j)}, k_{j}, k_{2}+K_{2}^{(i j)}\right),
$$

where we have made use of the notation $K_{1}^{\left(i_{1} \cdots i_{r}\right)}$ defined to be the sum of external momenta such that $K_{1}^{\left(i_{1} \cdots i_{r}\right)}+K_{2}^{\left(i_{1} \cdots i_{r}\right)}=k_{3}+\cdots+k_{2 n}-k_{i_{1}}-\cdots-k_{i_{r}}$. Using the $\epsilon$ expansion given in the appendix $\mathrm{D}$ and the symmetry of the coefficient in the exchange between $i$ and $j$, we can isolate the infra-red divergent part of this amplitude

$$
\begin{aligned}
\left.A_{2 n ; q}^{\text {one-loop }}\left(k_{1}^{-}, k_{2}^{-}, k_{3}^{+}, \cdots, k_{2 n}^{+}\right)\right|_{I R} & =\frac{2 r_{\Gamma} \mu^{2 \epsilon}}{\epsilon^{2}} \sum_{\substack{3 \leq i \neq j \leq 2 n \\
K_{1}^{(i j)}}} \tilde{c}_{4 ; k_{i}\left|k_{1}\right| k_{j} \mid k_{2}} \times \\
& \times\left(\left(-\left(k_{1}+k_{i}+K_{1}^{(i j)}\right)^{2}\right)^{-\epsilon}-\left(-\left(k_{1}+K_{1}^{(i j)}\right)^{2}\right)^{-\epsilon}\right) .
\end{aligned}
$$

The infra-red divergence associated with the multi-particle invariant $\left(k_{1}+K_{1}^{(i j)}\right)^{2}$ is given by

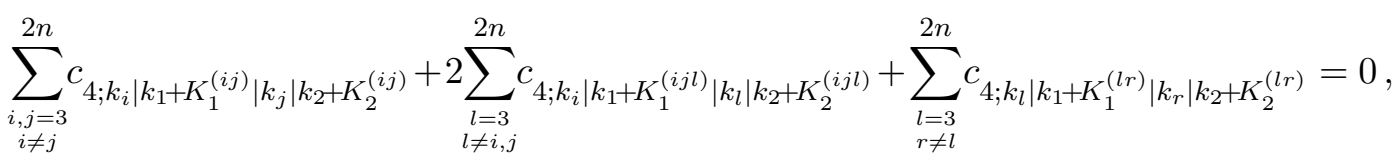

which shows that the amplitude is free of infra-red divergences, as it should be, since all the soft factors are vanishing for a multi-photon amplitude.

\section{Conclusions}

In this paper we have considered amplitudes in unordered field theories such as gravity and QED. New integral reduction formulæ derived using the world-line formalism have been examined and we have seen how such formulæ can have a wide range of applications in four dimensional theories.

It was shown in $[23,39]$ that, for maximal $\mathcal{N}=8$ supergravity, the constraints from the new integral reduction formulæ leads to the 'no-triangle' property for $n$-point supergravity 
amplitudes. In cases with less supersymmetry e.g. $\mathcal{N}=4$ supergravity it means that the $n$-graviton amplitude contains only integral functions up to scalar bubble integrals and that it is constructible from its cuts in $D=4-2 \epsilon[7,23,39]$. For pure gravity our result yields an amplitude consisting of scalar box, triangle and bubble integrals as well as rational polynomial terms. These results are completely surprising from naïve power counting arguments.

In this paper we have showed that one can apply the reduction formulæ eq. (2.30) and eq. (2.34) to the one-loop multi-photon amplitudes in QED to obtain that the oneloop multi-photon amplitudes with at least eight external photons are given by scalar box integral functions only. Such amplitudes satisfy a no-triangle property from $n \geq 8$ and are thus given solely by their quadruple cut. The amplitudes contain no rational polynomial contributions. This 'no triangle' property of multi-photon QED amplitude with $n \geq 8$ photons is true for helicity configurations of the external photons generalising the pure MHV results of Mahlon [38]. This result is clearly unexpected from naïve power counting arguments. Of course the appearance of the various scalar box integral functions in the expression for the amplitude depends on the helicity configuration of the external states. It would be interesting to reproduce these results for generic helicity configurations using a Wilson loop representation of the amplitude $[67,68]$.

We have shown that the considered cancellations can be made manifest by a choice of transverse gauge and the summation over the permutations of the unordered legs. We expect that unexpected cancellations should also appear in amplitudes with mixed photongluon external states. For such amplitudes one should expect a number of cancellations in the summation over the unordered photon lines.

Investigations of higher loop multi-photon and multi-graviton amplitudes presents another interesting direction for further investigation. Within the unitarity method formalism the cancellations seen for one-loop unordered amplitudes pose various restrictions on the type of integral functions appearing in the expansion of multi-loop amplitudes [20]. Factorisation based on 'no-triangle' properties at one-loop definitely suggest that amplitudes should have a simpler form (due to cancellations between orderings) than naïve counting proposes. For maximal $\mathcal{N}=8$ supergravity this gives a necessary (but not sufficient) requirement for the absence of the three-loop divergence in four dimensions [21].

The results of this paper show that a world-line approach is a particularly good framework for analysing the properties of loop amplitudes in unordered field theories. An extension of the world-line formalism to higher loops would be very helpful in this respect and would be required for a better understanding of the perturbative structure of $\mathcal{N}=8$ supergravity. This would help justifying and resolving the various constraints from supersymmetry [17] and dualities [18,19] in four dimensions and might lead to a conclusive argument for or against perturbative finiteness of maximal $\mathcal{N}=8$ supergravity in $D=4$.

\section{Acknowledgements}

We would like to thank Zvi Bern, David Kosower and Lance Dixon for many enlightening discussions. We are grateful to Lance Dixon for attracting our attention to the case of 
QED amplitudes. We would like to thank as well Zoltan Kuntz and Pierpaolo Mastrolia for comments on the draft and Gregory Korchemsky for discussions. This research was supported in part (NEJBB) by grant DE-FG0290ER40542 of the US Department of Energy and (PV) by the RTN contracts MRTN-CT-2004-503369, MRTN-CT-2004-005104, as well as the "Agence Nationale de la Recherche" grants BLAN06-3-137168 and ANR-05-BLAN0073-01. SB also acknowledges support from the Helmholtz Gemeinschaft under contract VH-NG-105.

\section{A. Helicity formalism conventions}

All conventions and the notation in the paper follows that of ref. [56] unless otherwise stated.

We will here employ the mostly minus metric signature $\eta^{\mu \nu}=\operatorname{diag}(+,-,-,-)$ and use a representation of the Dirac matrices satisfying $\left\{\gamma^{\mu}, \gamma^{\nu}\right\}=2 \eta^{\mu \nu}$, i.e.,

$$
\gamma^{\mu}=\left(\begin{array}{cc}
0 & \sigma^{i} \\
-\sigma^{i} & 0
\end{array}\right) ; \quad \gamma_{5}=\left(\begin{array}{cc}
1 & 0 \\
0 & -1
\end{array}\right)
$$

Here $\left(\sigma^{\mu}\right)=\left(1, \sigma^{i}\right)$ and $\left(\bar{\sigma}^{\mu}\right)=\left(-1, \bar{\sigma}^{i}\right)$ and $\sigma^{i}$ are the standard Pauli matrices. We will make use of the slashed notation $\gamma^{\mu} p_{\mu}=\not p$.

For any light-like momentum $p$ the positive energy solution to the Dirac equation is $\not p u_{h}(p)=0$ both for positive and negative helicities, i.e., $h=+1$ and $h=-1$. This solution satisfy the chirality condition $\left(1 \pm \gamma_{5}\right) / 2 u_{ \pm}(p)=0$ and $\left(1 \mp \gamma_{5}\right) / 2 u_{ \pm}(p)=0$.

We will make use of the following conventions

$$
\begin{aligned}
|k\rangle \equiv u_{+}(k) ; & \mid k] \equiv u_{-}(k) \\
\langle k| \equiv \bar{u}_{-}(k) ; & {\left[k \mid \equiv \bar{u}_{+}(k) .\right.}
\end{aligned}
$$

Spinor products will be defined according to

$$
\langle p q\rangle \equiv \bar{u}_{-}(p) u_{+}(q) ; \quad[p q] \equiv \bar{u}_{+}(p) u_{-}(q)
$$

where $(p+q)^{2}=2 p \cdot q=\langle p q\rangle[p q]$.

With these conventions the completeness relation gives that

$$
\sum_{h= \pm 1} u_{h}(k) \bar{u}_{h}(k)=\not k=|k\rangle[k|+| k]\langle k|
$$

The polarisation tensor for the photon of light-like momentum $k$ can represented as

$$
\notin^{+}\left(k, p_{\text {ref }}\right)=\frac{\left|p_{\text {ref }}\right\rangle[k \mid}{\sqrt{2}\left\langle p_{\text {ref }} k\right\rangle} ; \quad \notin^{-}\left(k, p_{\text {ref }}\right)=-\frac{\left.\mid p_{\text {ref }}\right]\langle k|}{\sqrt{2}\left[p_{\text {ref }} k\right]},
$$

where $p_{\text {ref }}$ is an arbitrary light-like reference momentum. 


\section{B. Proof of the improved large $z$ behaviour of the $e^{-} e^{+} \rightarrow n \gamma$ tree-amplitude}

In this section we will prove the large $z$ behaviour of the $e^{+} e^{-} \rightarrow n \gamma$ tree-level amplitude under the BCFW shifts, see (3.19) and (3.20). The behaviour of the scalar amplitude can be related to that of the fermion by supersymmetric Ward identities.

We will first write the amplitude with $n$ external photons in the following way

$$
A_{n ; q}^{\text {tree }}\left(p_{a}^{h}, p_{b}^{-h} ; k_{1}, \ldots, k_{n}\right)=\sum_{i=1}^{n} \sum_{\sigma \in \mathfrak{S}_{n-1}}(-1)^{n-i} \frac{e}{n} \bar{u}_{h}\left(p_{a}\right) \mathfrak{J}_{1 \cdots \hat{\imath} \cdots n}^{\text {tree }} \frac{\not p_{b}+\not k_{i}}{\left(p_{b}+k_{i}\right)^{2}} \notin_{i} u_{-h}\left(p_{b}\right) \text {. }
$$

Where $\mathfrak{J}_{1 \cdots \hat{\imath} \cdots n}^{\text {tree }}$ is an off-shell current constructed from the remaining $n-1$ photons after the $i^{\text {th }}$ photon is removed from the list. We have

$$
\mathfrak{J}_{1 \cdots \hat{\imath} \cdots n}^{\mathrm{tree}} \equiv \phi_{1} \frac{i \not q_{1}}{q_{1}^{2}} \cdots \phi_{i-1} \frac{i \not q_{i-1}}{q_{i-1}^{2}} \phi_{i+1} \frac{i \not q_{i+1}}{q_{i+1}^{2}} \cdots \frac{i \not \not_{n-1}}{q_{n-1}^{2}} \notin_{n}
$$

Here $q_{j}=p_{a}+k_{1}+\cdots+k_{j}$ where, as before, we have not included the momentum of the $i^{\text {th }}$ state. Using that $\not p_{b} \notin_{i}+\notin_{i} \not p_{b}=2 p_{b} \cdot \epsilon_{i}$ and $\not p_{b} u_{-h}\left(p_{b}\right)=0$ we rewrite

$$
\not p_{b} \notin_{i} u_{-h}\left(p_{b}\right)=2 u_{-h}\left(p_{b}\right) p_{b} \cdot \epsilon_{i}
$$

Choosing the reference momentum of the photons to be $p_{\text {ref }}=p_{b}$ so that $\epsilon_{i} \cdot p_{b}=0$ one can rewrite the tree-amplitude as

$$
A_{n ; q}^{\text {tree }}\left(p_{a}^{h}, p_{b}^{-h} ; k_{1}, \ldots, k_{n}\right)=\sum_{i=1}^{n} \sum_{\sigma \in \mathfrak{S}_{n-1}}(-1)^{n-i} \frac{e}{n} \frac{\left.\bar{u}_{h}\left(p_{a}\right)\right)_{\mathfrak{J}_{1 \cdots \hat{\imath} \cdots n}}^{\text {tree }} \not k_{i} \notin_{i} u_{-h}\left(p_{b}\right)}{\left(p_{b}+k_{i}\right)^{2}} .
$$

With this choice of reference momentum we also have that

$$
\phi_{i}^{-} u_{-}\left(p_{b}\right)=0, \quad \phi_{i}^{+} u_{+}\left(p_{b}\right)=0 .
$$

Only the non-zero contributions are such that the helicity of the $i^{\text {th }}$ photon is the opposite of the one of the positron. We remark as well that

$$
\frac{\not \ell_{i} \notin_{i}^{h} u_{-h}\left(p_{b}\right)}{\left(p_{b}+k_{i}\right)^{2}}=\frac{\not k_{i} u_{-h}\left(p_{b}\right)}{\sqrt{2}\left(p_{b}+k_{i}\right)^{2}}=\frac{u_{-h}\left(k_{i}\right)}{\sqrt{2}\left\langle k_{i}^{h} \mid p_{b}^{-h}\right\rangle} .
$$

Combining these properties we arrive at the following expression for the tree-level amplitude

$$
A_{n ; q}^{\text {tree }}\left(p_{a}^{h}, p_{b}^{-h} ; k_{1}, \ldots, k_{n}\right)=\sum_{i=1}^{n} \sum_{\sigma \in \mathfrak{S}_{n-1}}(-1)^{n-i} \delta\left(h_{i}+h=0\right) \frac{e}{n} \frac{\bar{u}_{h}\left(p_{a}\right) \mathfrak{J}_{1 \cdots \hat{\imath} \cdots n}^{\text {tree }} u_{-h}\left(k_{i}\right)}{\sqrt{2}\left\langle k_{i}^{-h} \mid p_{b}^{h}\right\rangle} .
$$

This means that the multi-photon tree-level amplitude has been rewritten as a sum of $(n-1)$-photon off-shell currents, $\mathfrak{J}_{1 \cdots \hat{\imath} \cdots n}^{\text {tree }}$. All external photons in this expression have $p_{b}$ as their reference momentum. We can now study the large $z$ behaviour of the tree-level amplitude $A_{n ; q}^{\text {tree }}$ under the BCFW shifts (3.19) and (3.20). 
Because the reference momentum of the photons is $p_{b}$ we have that for each polarisation choice, in the limit where $z \rightarrow \infty$, that the polarisation tensor behave as

$$
\phi_{\infty}^{+}\left(p_{b}, k\right)=\lim _{z \rightarrow \infty} \AA^{+}\left(\widehat{p}_{b}, k\right)=\lim _{z \rightarrow \infty} \frac{\left|\widehat{p}_{b}\right\rangle[k \mid}{\left\langle\widehat{p}_{b} k\right\rangle}=\frac{s+1}{2} \frac{\left|p_{b}\right\rangle[k \mid}{\left\langle p_{b} k\right\rangle}+\frac{s-1}{2} \frac{\left|p_{a}\right\rangle[k \mid}{\left\langle p_{a} k\right\rangle},
$$

and

$$
\notin_{\infty}^{-}\left(p_{b}, k\right)=\lim _{z \rightarrow \infty} \notin^{-}\left(\widehat{p}_{b}, k\right)=\lim _{z \rightarrow \infty} \frac{\left.\mid \widehat{p}_{b}\right]\langle k|}{\left[\widehat{p}_{b} k\right]}=\frac{s+1}{2} \frac{\left.\mid p_{a}\right]\langle k|}{\left[p_{a} k\right]}+\frac{s-1}{2} \frac{\left.\mid p_{b}\right]\langle k|}{\left[p_{b} k\right]}
$$

which is independent of $z$.

We will now consider the behaviour of the product of a fermion propagator and a polarisation

$$
T_{i}=\phi^{ \pm}\left(\widehat{p}_{b}, k_{i}\right) \frac{\widehat{q}_{i}}{\widehat{q}_{i}^{2}} .
$$

Under the BCFW shift the momentum $\widehat{q}_{i}$ shift according eq. (3.21)

$$
\widehat{q}_{i}=q_{i}+z \not t
$$

where $\not$ is defined in eq. (3.22)

$$
\star \equiv \frac{1+s}{2}\left(\left|p_{b}\right\rangle\left[p_{a}|+| p_{a}\right]\left\langle p_{b}\right|\right)+\frac{s-1}{2}\left(\left|p_{a}\right\rangle\left[p_{b}|+| p_{b}\right]\left\langle p_{a}\right|\right) .
$$

Since

$$
\lim _{z \rightarrow \infty} \notin^{ \pm}\left(\widehat{p}_{b}, k_{i}\right) \pitchfork=0
$$

we conclude that

$$
\lim _{z \rightarrow \infty} \phi^{ \pm}\left(\widehat{p}_{b}, k_{i}\right) \widehat{q}_{i}=\phi_{\infty}^{ \pm}\left(\widehat{p}_{b}, k_{i+1}\right) \not_{i},
$$

which is independent of $z$. Therefore the quantity $T_{i}$ has the large $z$ behaviour

$$
\lim _{z \rightarrow \infty} T_{i} \sim \frac{1}{z} \frac{\phi_{\infty}^{ \pm}\left(\widehat{p}_{b}, k_{i}\right) \not q_{i}}{2\left(q_{i} \cdot \pi\right)}
$$

We now consider the two ends of the amplitude $A_{n ; q}^{\text {tree }}$ written in the form of (B.7)

$$
T_{a}=\bar{u}_{h}\left(\widehat{p}_{a}\right), \quad T_{n-1}=\frac{\notin^{ \pm}\left(p_{b}, k_{n}\right) u_{-h}\left(k_{i}\right)}{\left\langle k_{i}^{-h} \mid \widehat{p}_{b}^{h}\right\rangle} .
$$

Clearly $T_{n}$ has the large $z$ behaviour given by

$$
\lim _{z \rightarrow \infty} T_{n}=\lim _{z \rightarrow \infty} \frac{\notin^{ \pm}\left(p_{b}, k_{n}\right) u_{-h}\left(k_{i}\right)}{\left\langle k_{i}^{-2 h} \mid p_{b}^{2 h}\right\rangle} \sim z^{h s-1 / 2} \notin_{\infty}^{ \pm}\left(p_{b}, k_{n}\right) u_{-h}\left(k_{i}\right) \frac{\left\langle k_{i}^{h} \mid p_{b}^{-h}\right\rangle}{2 \pi \cdot k_{i}} ; \quad h= \pm \frac{1}{2} .
$$

For $T_{a}$ (by definition of the shift on the fermion polarisations) we have

$$
\lim _{z \rightarrow \infty} T_{a} \sim z^{h s+1 / 2} ; \quad h= \pm \frac{1}{2} .
$$


Collecting all the terms we conclude that each term in the sum (B.7)

$$
\frac{\bar{u}_{h}\left(p_{a}\right) \tilde{J}_{1 \cdots \hat{\imath} \cdots n}^{\text {tree }} u_{-h}\left(k_{i}\right)}{\left\langle k_{i}^{-2 h} \mid p_{b}^{2 h}\right\rangle}=T_{a} T_{1} \cdots T_{i-1} T_{i+1} \cdots T_{n-1} T_{n}
$$

behaves as

$$
\lim _{z \rightarrow \infty} \frac{\bar{u}_{h}\left(p_{a}\right) \mathfrak{J}_{1 \cdots \hat{\imath} \cdots n}^{\text {tree }} u_{-h}\left(k_{i}\right)}{\left\langle k_{i}^{-2 h} \mid p_{b}^{2 h}\right\rangle} \sim z^{2 h s} \frac{1}{z^{n-2}} ; \quad h= \pm \frac{1}{2} .
$$

This concludes the proof of the large $z$ scaling property of the $e^{+} e^{-} \rightarrow n \gamma$ tree-level amplitude under the BCFW shifts (3.19) and (3.20).

The analysis for the fermion case in QED is rather special because the fermions can only be adjacent in the interactions that are involved. This is however not the case for generic amplitudes for example in gravity. It should be noted that while the choice of reference momentum $p_{\text {ref }}=p_{b}$ was enough for obtaining the required scaling and that no cancellations between terms of different orderings were necessary for the result it is easy to verify that other choices of reference momentum would require cancellations between terms of different orderings in order to arrive at the large $z$ scaling result eq. (B.20).

Using supersymmetric Ward identities we can conclude that the massless scalar tree amplitudes have the large $z$ scaling given by

$$
\lim _{z \rightarrow \infty} A_{n ; \varphi}^{\text {tree }} \sim \frac{1}{z^{n-2}} .
$$

In the scalar case as explained in section 3.3.3 a gauge choice is not enough for obtaining this behaviour and extra cancellations has to arise in the sum over orderings.

\section{Cut analysis of one-loop $n$-photon integral coefficients}

In this appendix we will give further details regarding the tree-level $z$-scaling, eq. (3.23) of the amplitude. The knowledge of the tree amplitudes BCFW $z$-scaling behaviour combined with an analysis of unitarity cuts for example using a formalism such as Forde [43] have been used successfully to show analogous simplifications in gravity theories see refs. $[6,7]$. For demonstrating the absence of scalar triangle and bubble integrals in the one-loop $n$ photon amplitude for $n \geq 6$ this is a very powerful strategy. To examine the analytic structure of the rational terms we use $D$-dimensional cutting techniques $[41,42,45,60,61]$. We will prove the vanishing of rational polynomial terms in the QED amplitudes using a generalisation of Forde's method for $D$-dimensional cuts, re-expressed in terms of massive four-dimensional cuts [47].

\section{C.1 Absence of triangles in amplitudes with $n>6$}

We will first analyse the presence of triangles in the $n$-photon one-loop amplitude, by considering the triple cut represented in figure 5(b)

$$
\left.A_{n ; q}^{\text {one-loop }}\right|_{3 \text {-cut }}=(2 \pi)^{3} \int d^{4} \ell \prod_{i=1}^{3} \delta\left(\ell_{i}^{2}\right) \sum_{h= \pm \frac{1}{2}} A_{n_{1}}^{\text {tree }}\left(\ell_{1}^{2 h},-\ell_{2}^{-2 h}\right) A_{n_{2}}^{\text {tree }}\left(\ell_{2}^{2 h},-\ell_{3}^{-2 h}\right) A_{n_{3}}^{\text {tree }}\left(\ell_{3}^{2 h},-\ell_{1}^{-2 h}\right) \text {. }
$$




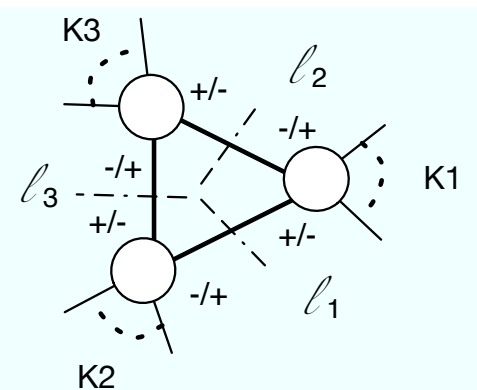

Figure 6: The triple-cut contribution the amplitude.

Here $n_{1}+n_{2}+n_{3}=n$. We have only indicated the momenta of the fermions, which are given by the cut loop momenta. Following [43] we can parametrise the loop momenta $\ell_{i}$ with $i=1,2,3$ in the cut as

$$
\left.\ell_{i}=t \mid K_{1}^{b}\right]\left\langle K_{2}^{b}\left|+\frac{\alpha_{i 1} \alpha_{i 2}}{t}\right| K_{1}^{b}\right\rangle\left[K_{2}^{b} \mid+\alpha_{i 1} K_{2}^{b}+\alpha_{i 2} K_{1}^{b},\right.
$$

where $\ell=\ell_{3}$. The shifted propagator factors behaves as

$$
\left.\lim _{t \rightarrow \infty} \widehat{\not ̆}_{i} \sim t \mid K_{1}^{b}\right]\left\langle K_{2}^{b}\right| \text {. }
$$

With this parametrisation of the loop momenta the polarisations of the fermions will be given by

$$
\begin{aligned}
& \left.\left.\left.u_{-}\left(\ell_{i}\right) \equiv \mid \ell_{i}\right]=t \mid K_{1}^{b}\right]+\alpha_{i 1} \mid K_{2}^{b}\right] \\
& u_{+}\left(\ell_{i}\right) \equiv\left|\ell_{i}\right\rangle=\frac{\alpha_{i 2}}{t}\left|K_{1}^{b}\right\rangle+\left|K_{2}^{b}\right\rangle,
\end{aligned}
$$

which have the large $t$ behaviour

$$
\left.\lim _{t \rightarrow \infty} u_{+}\left(\ell_{i}\right) \sim t\left|K_{1}^{b}\right\rangle, \quad \lim _{t \rightarrow \infty} u_{-}\left(\ell_{i}\right) \sim \mid K_{2}^{b}\right]
$$

The large $t$ scaling is equivalent to the BCFW shift scaling of type $s=+1$ in (3.19) (except for the scaling of the $u_{-}\left(\ell_{i}\right)$ which scales like $z$ in (3.21) $)$. Because of this the amplitude will have only less power of $t$ in the numerator for the external fermion line. Of course for the scalar tree-level amplitude there is no $t$ factor from the external scalar states.

Taking into account the scaling of the external states we can conclude (using the found large $z$ scaling of the tree amplitudes given in (3.23) ) that the large $t$ behaviour is

$$
\lim _{t \rightarrow \infty} A_{n}^{\text {tree }}\left(\ell_{i}^{2 h},-\ell_{j}^{-2 h}, k_{1}, \ldots, k_{n}\right) \sim t^{2-n} .
$$

We remark that this behaviour is independent of the helicity $h$ of the state running in the loop. Therefore the large $t$ behaviour of the cut (C.1) is given by

$$
\lim _{t \rightarrow \infty} A_{n_{1}}^{\text {tree }}\left(\ell_{1}^{2 h},-\ell_{2}^{-2 h}\right) A_{n_{2}}^{\text {tree }}\left(\ell_{2}^{2 h},-\ell_{3}^{-2 h}\right) A_{n_{3}}^{\text {tree }}\left(\ell_{3}^{2 h},-\ell_{1}^{-2 h}\right) \sim \frac{1}{t^{n-6}} .
$$

We can thus conclude that the one-loop $n>6$ photon amplitude with either a fermion or a scalar running in the loop do not contain scalar triangle integrals with massive corners. 


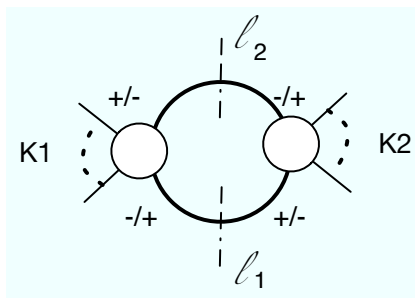

(a)

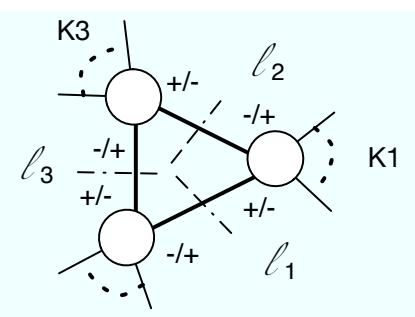

(b)

Figure 7: The double-cut contribution to the amplitude is given by (a) the bubble cut and (b) the triangle subtraction.

\section{C.2 Absence of bubbles in amplitudes with $n>4$}

In this section we examine the two-line cut of the amplitude.

The cut amplitude can be computed as

$$
\left.A_{n ; q}^{\text {one-loop }}\right|_{2 \text {-cut }}=(2 \pi)^{3} \int d^{4} \ell_{1} \delta\left(\ell_{1}^{2}\right) \delta\left(\ell_{2}^{2}\right) \sum_{h= \pm \frac{1}{2}} A_{n_{1}}^{\text {tree }}\left(\ell_{1}^{2 h},-\ell_{2}^{-2 h}\right) A_{n_{2}}^{\text {tree }}\left(\ell_{2}^{2 h},-\ell_{1}^{-2 h}\right),
$$

with $n_{1}+n_{2}=n$. The cut loop momenta can be parametrised as follows [43]

$$
\left.\left.\left.\mid l_{1}\right]=t \mid K_{1}^{b}\right]+(1-y) \frac{K_{1}^{2}}{\gamma} \mid \chi^{-}\right], \quad\left|l_{1}\right\rangle=\frac{y}{t}\left|K_{1}^{b}\right\rangle+|\chi\rangle
$$

and

$$
\left.\left.\left.\mid l_{2}\right]=\mid K_{1}^{b}\right]-\frac{y}{t} \frac{K_{1}^{2}}{\gamma} \mid \chi\right], \quad\left|l_{2}\right\rangle=(y-1)\left|K_{1}^{b}\right\rangle+t|\chi\rangle .
$$

For large $t$ with $t \gg y$, the behaviour is like the one of the triangle cut analysed in the previous section. The analysis in the previous section showed that no triangles are present in amplitudes with $n \geq 7$ photons, so for these amplitudes the leading behaviour of the amplitude for $y \gg t \gg 1$ will be a test for possible bubble contributions. For $n=6$ the large $t$ behaviour of the triple-cut amplitude is given by a constant. In that case as well the leading behaviour of the amplitude for $y \gg t \gg 1$ will be enough for analysing the bubble contributions. For $n \leq 5$ one has to pay attention to the triangle subtractions [43] represented in fig. 7 that can lead to a contribution in the regime where $y \sim t$. We will discuss these contributions below.

For analysing the presence of scalar bubble integrals one needs to take the limits $y \rightarrow \infty, t \rightarrow \infty$ and $y \gg t \gg 1$. In this case the polarisation of the fermion shifts according to

$$
\begin{aligned}
u_{-}\left(\ell_{1}\right) & \left.\sim-y \frac{K_{1}^{2}}{\gamma} \mid \chi\right], \quad u_{+}\left(\ell_{1}\right) \sim \frac{y}{t}\left|K_{1}^{b}\right\rangle, \\
u_{-}\left(\ell_{2}\right) & \left.\sim-\frac{y}{t} \mid K_{1}^{b}\right], \quad u_{+}\left(\ell_{2}\right) \sim y\left|K_{1}^{b}\right\rangle .
\end{aligned}
$$

The polarisations of the scalar fields do not shift. The shifted propagator factors behaves as

$$
\left.\lim _{t \rightarrow \infty} \widehat{\not}_{i} \sim-\frac{y^{2}}{t} \frac{K_{1}^{2}}{2 \gamma} \mid K_{1}^{b}\right]\langle\chi|
$$


From the analysis of the large $z$ behaviour of the BCFW shift in section 3.3 we conclude that the large $y \gg t \gg 1$ of the tree amplitudes behave as

$$
\begin{aligned}
A_{n_{1}}^{\text {tree }}\left(\ell_{1}^{-2 h},-\ell_{2}^{+2 h}\right) & \sim\left(\frac{y^{2}}{t}\right)^{2-n_{1}} t^{2 h}, \\
A_{n_{2}}^{\text {tree }}\left(\ell_{2}^{-2 h},-\ell_{1}^{+2 h}\right) & \sim\left(\frac{y^{2}}{t}\right)^{2-n_{2}} t^{-2 h},
\end{aligned}
$$

for $h= \pm \frac{1}{2}$ for the fermion loop and $h=0$ for the scalar loop. And the integrand of the cut amplitude in (C.8) behaves as

$$
A_{n_{1}}^{\text {tree }}\left(\ell_{1}^{2 h},-\ell_{2}^{-2 h}\right) A_{n_{2}}^{\text {tree }}\left(\ell_{2}^{2 h},-\ell_{1}^{-2 h}\right) \sim\left(\frac{y^{2}}{t}\right)^{4-n} .
$$

The answer is independent of the helicity and of the nature of the particle (fermionic or scalar) running in the loop.

From this scaling we can conclude that no bubbles appear in amplitudes with $n \geq 6$ photons. Furthermore the amplitude with four photons contains bubble contributions as directly confirmed by the explicit evaluation of the amplitude [70,71].

For $n=5$ photons the leading behaviour in (C.14) vanishes and no pure scalar bubble contributions are found in the amplitude. In this case there are non-vanishing triangle subtractions from the regime where $y \sim t$ as represented in fig. 1. All amplitudes vanishes via Furry's theorems and the triangles contributions add up to zero via symmetry properties of the amplitude (3.5) and after summing over the orderings of external legs.

\section{C.3 Absence of rational terms}

In this section we connect the large $z$ behaviour (3.29) of the massive scalar tree-amplitude to the large $\mu^{2}$ limit of massive scalar loop amplitudes for testing for rational terms contributions following the method used in $[43,47]$. In this section we will follow closely the notations and conventions of ref. [47]. The analysis makes use of the $D$-dimensional integral basis recently used in numerical implementations of the $D$-dimensional unitarity method [45].

To analyse the rational contributions to the $n$-photon loop amplitudes we need to consider $D$-dimensional unitarity cuts. We can relate the $D$-dimensional loop momenta to massive momentum using:

$$
\ell_{[D]}^{\nu}=\bar{\ell}^{\nu}+\ell_{[-2 \epsilon]}^{\nu}, \quad \bar{\ell}^{2}=\mu^{2} .
$$

Using a $D$-dimensional integral basis it is possible to write the rational contributions in a basis of massive box, triangle and bubble functions by relating the $D$-dimensional integral to integrals of the mass parameter, $\mu$. This results in [45]:

$$
R_{n}=\sum_{K_{4}} C_{4 ; K_{4}}^{[4]} I_{4 ; K_{4}}\left[\mu^{4}\right]+\sum_{K_{3}} C_{3 ; K_{3}}^{[2]} I_{3 ; K_{3}}\left[\mu^{2}\right]+\sum_{K_{2}} C_{2 ; K_{2}}^{[2]} I_{2 ; K_{2}}\left[\mu^{2}\right]
$$

where $K_{i}$ with $1 \leq i \leq 4$ denote the set of momenta of the massive scalar box integral functions. 
After performing the $\bar{\ell} \sim \mu$ loop momentum integration and taking the $\epsilon \rightarrow 0$ limit this becomes [45]:

$$
R_{n}=-\frac{1}{6} \sum_{K_{4}} C_{4 ; K_{4}}^{[4]}-\frac{1}{2} \sum_{K_{3}} C_{3 ; K_{3}}^{[2]}-\frac{1}{6} \sum_{K_{2}} C_{2 ; K_{2}}^{[2]} K_{2}^{2} .
$$

The coefficients $C_{4 ; K_{4}}^{[4]}, C_{3 ; K_{3}}^{[2]}$ and $C_{2 ; K_{2}}^{[2]}$ can then be extracted from the analysis of the large momentum scaling of the generalised cuts with four dimensional massive propagators.

For multi-photon loop amplitudes the rational terms can be extracted from tree amplitudes with a massive fermion in the loop. The use of massive fermions is however delicate to use in amplitudes evaluated in $D=4-2 \epsilon$. Therefore we will use the supersymmetric decomposition to write, the QED amplitude as the one-loop amplitude for $\mathcal{N}=1$ super-QED minus the contributions of a scalar loop

$$
A_{n ; q}^{\text {one-loop }}=A_{n ; \mathcal{N}=1}^{\text {one-loop }}-A_{n ; \varphi}^{\text {one-loop }} .
$$

Since any supersymmetric amplitudes are cut constructible in four dimensions, all the rational pieces are arising from the scalar loop contribution. The rational part contribution to a scalar amplitude can be extracted by introducing an effective mass $\mu^{2}$ for the scalar and by evaluating the integral coefficient in four dimensions with the tree-level amplitudes for massive scalars [74-76].

\section{$\mu^{2}$ Dependence Of The Box Coefficients}

For the quadruple cut of the massive scalar loop multi-photon amplitude we choose the following basis for the loop momentum (see section 4.1 of [47] for notation),

$$
\bar{\ell}_{1}=a K_{4}^{b}+b K_{1}^{b}+c\left|K_{4}^{b}\right\rangle\left[K_{1}^{b}\left|+\frac{\gamma_{14} a b-\mu^{2}}{\gamma_{14} c}\right| K_{1}^{b}\right\rangle\left[K_{4}^{b} \mid,\right.
$$

where the on-shell constraints determine

$$
\begin{aligned}
\gamma_{14} & =K_{1} \cdot K_{4} \pm \sqrt{K_{1} \cdot K_{4}-K_{1}^{2} K_{4}^{2}}, \\
a & =\frac{K_{1}^{2}\left(K_{4}^{2}+\gamma_{14}\right)}{\gamma_{14}^{2}-K_{1}^{2} K_{4}^{2}} ; \quad b=\frac{K_{4}^{2}\left(K_{1}^{2}+\gamma_{14}\right)}{\gamma_{14}^{2}-K_{1}^{2} K_{4}^{2}},
\end{aligned}
$$

which do not depend on $\mu$, and two solutions for $c=c_{ \pm}$that have the large $\mu$ limit

$$
\lim _{\mu^{2} \rightarrow \infty} c_{ \pm}= \pm|\mu| \sqrt{\frac{\left\langle K_{1}^{b}\left|K_{2}\right| K_{4}^{b}\right]}{\gamma_{14}\left\langle K_{4}^{b}\left|K_{2}\right| K_{1}^{b}\right]}} .
$$

The box-type rational contribution is given by the large $\mu$ behaviour of the quadruple cut:

$$
C_{4}^{[4]}=\left.\frac{i}{2} \sum_{c=c \pm} \inf _{\mu^{2}}\left[A_{n_{1}}^{\text {tree }} A_{n_{2}}^{\text {tree }} A_{n_{3}}^{\text {tree }} A_{n_{4}}^{\text {tree }}\left(\bar{\ell}_{1}(c)\right)\right]\right|_{\mu^{4}}
$$

with $n_{1}+n_{2}+n_{3}+n_{4}=n$. For a function $f(x)$ with at most a polynomial growth for $x \rightarrow \infty$

$$
\lim _{x \rightarrow \infty} f(x)=a_{n} x^{n}+\cdots+a_{0}+\mathcal{O}(1 / x)
$$


we define the 'inf' operation following [77]

$$
\inf _{x} f=a_{n} x^{n}+\cdots+a_{0} .
$$

We will use as well the notation $\left.\inf _{x} f\right|_{x^{k}}=a_{k}$ for the coefficient of $x^{k}$.

To see the cancellation of such terms in the photon amplitudes we use the scaling of a generic tree amplitude in the regime of large $\mu$ derived in section 3.2.2.

For large $\mu$, since $c \sim \mu$ the loop momenta in the cuts scale as

$$
\lim _{\mu^{2} \rightarrow \infty} \bar{\ell}_{1}(c) \sim \mu \chi
$$

where $\chi$ is some non-vanishing vector, which is the behaviour of eq. (3.32), and the analysis of the end of section 3.2 .2 gives that the massive scalar tree-level amplitude has the behaviour

$$
\lim _{\mu^{2} \rightarrow \infty} A_{n}^{\text {tree }}=\frac{1}{\mu^{n-2}} .
$$

This implies that the product of the four tree-level factor in (․23) has the large $\mu$ behaviour

$$
\lim _{\mu^{2} \rightarrow \infty} A_{n_{1}}^{\text {tree }} A_{n_{2}}^{\text {tree }} A_{n_{3}}^{\text {tree }} A_{n_{4}}^{\text {tree }}\left(\bar{\ell}_{1}(c)\right) \sim \frac{\mu^{4}}{\mu^{n-4}}
$$

implying that $C_{4}^{[4]}\left(k_{1}, \cdots, k_{4}\right)$ does not vanish for the four-photon amplitudes which hence will receive a contribution from rational pieces in agreement with the explicit computation in $[38,70,71]$. For more than four photons the one-loop amplitude does not have any rational term contribution

$$
C_{4}^{[4]}\left(k_{1}, \ldots, k_{n}\right)=0 \quad \text { for } n>4 .
$$

\section{$\mu^{2}$ Dependence Of The Triangle Coefficients}

For the triple cut of the massive scalar loop multi-photon amplitude we choose the following basis for the loop momentum (see section 4.2 of [47])

$$
\bar{\ell}_{1}=a K_{4}^{b}+b K_{1}^{b}+t\left|K_{4}^{b}\right\rangle\left[K_{1}^{b}\left|+\frac{\gamma_{14} a b-\mu^{2}}{\gamma_{14} t}\right| K_{1}^{b}\right\rangle\left[K_{4}^{b} \mid,\right.
$$

with the same expressions for $\gamma_{14}$ and $a$ and $b$ as in the previous section. In general there will be two solutions to the on-shell constraints $\bar{\ell}_{1}^{2}=0$ which we label $\bar{\ell}_{1}^{ \pm}$. We have

$$
C_{3}^{[2]}=\left.\frac{1}{2} \sum_{\sigma= \pm} \inf _{\mu^{2}}\left[\inf _{t}\left[A_{n_{1}}^{\text {tree }} A_{n_{2}}^{\text {tree }} A_{n_{3}}^{\text {tree }}\left(\bar{\ell}_{1}^{\sigma}\right)\right]\right]\right|_{t^{0}, \mu^{2}},
$$

with $n_{1}+n_{2}+n_{3}=n$. We must consider the product of three tree amplitudes in the $t \gg \mu \rightarrow \infty$ limit. In this limit the loop momenta takes the following asymptotic form

$$
\lim _{\mu^{2} \rightarrow \infty} \lim _{t \rightarrow \infty} \bar{y}_{1} \sim t\left|K_{4}^{b}\right\rangle\left[K_{1}^{b}\left|-\frac{\mu^{2}}{t} \frac{1}{\gamma_{14}}\right| K_{1}^{b}\right\rangle\left[K_{4}^{b} \mid .\right.
$$


Following the analysis of the triangle cut in section C.1 we deduce that in this limit the massive scalar tree amplitude scale like

$$
\lim _{t \rightarrow \infty} A_{n ; \varphi}^{\text {tree }} \sim \frac{1}{t^{n-2}}\left(C_{n ; \varphi}^{\infty}+\frac{\mu^{2}}{t^{2}} \delta C_{n ; \varphi}^{\infty}+\mathcal{O}\left(\mu^{4} / t^{4}\right)\right)
$$

The sub-leading corrections in $\mu^{2}$ arises from the $\mu^{2} / t$ dependence in (C.32) and because $\mu^{2}$ is dimensionful these corrections appear with a factor of order $1 / \bar{\ell}^{2}$ so that the corrections are of order $\mathcal{O}\left(\left(\mu^{2} / t\right) \times 1 / \bar{\ell}^{2}\right) \sim \mathcal{O}\left(\mu^{2} / t^{2}\right)$ in the large $t$ limit where $t \gg \mu^{2}$.

For $n>6$ the product of the tree amplitude hence behave as $O\left(1 / t^{n-6}\right)$ therefore

$$
\inf _{t}\left[A_{n_{1}}^{\text {tree }} A_{n_{2}}^{\text {tree }} A_{n_{3}}^{\text {tree }}\left(\bar{\ell}_{1}^{\sigma}\right)\right]=0
$$

so we can conclude that $C_{3}^{[2]}=0$ for the one-loop amplitude with $n>6$ external photons. For $n=6$ we have for each triangle contribution

$$
\left.\inf _{t}\left[C_{3}^{N_{3}-\text { mass }}\right]\right|_{t^{0}}=\frac{1}{2} \sum_{\sigma= \pm} C_{n_{1} ; \varphi}^{\infty} C_{n_{2} ; \varphi}^{\infty} C_{n_{2} ; \varphi}^{\infty}\left(\bar{\ell}_{1}^{\sigma}\right) ; \quad N_{3}=1,2,3 .
$$

The leading $\mu^{2}$ contribution does not depend on $\mu^{2}$ and there is no rational term contribution from scalar triangle integrals for the $n=6$ photon amplitude. The sub-leading contributions to the tree-amplitude are of order $\mathcal{O}\left(\mu^{2} / t^{n-1}\right)$. This imply that the contribution to the triple cut has the large $t$ expansion

$$
\inf _{t}\left[C_{3}^{N_{3}-\text { mass }}\right]=\inf _{t}\left[\frac{\mu^{2}}{t^{2}} \delta C_{n_{1} ; \varphi}^{\infty} C_{n_{2} ; \varphi}^{\infty} C_{n_{3} ; \varphi}^{\infty}\right]=0 ; \quad N_{3}=1,2,3,
$$

and hence there is no rational term contribution from triangles for $n=6$ photons.

Only the $n=4$ photon amplitude can get a contribution from the one-mass triangle. The product of the tree amplitudes leads to a $\mu^{2}$ contribution

$$
\begin{aligned}
\left.\inf _{t}\left[C_{3}^{1-\text { mass }}\right]\right|_{t^{0}} & =\left.\inf _{t}\left[t^{2} \prod_{i=1}^{3}\left(C_{n_{i} ; \varphi}^{\infty}+\frac{\mu^{2}}{t^{2}} \delta C_{n_{i} ; \varphi}^{\infty}\right)\right]\right|_{t=0} \\
& =\mu^{2}\left(C_{n_{1} ; \varphi}^{\infty} C_{n_{2} ; \varphi}^{\infty} \delta C_{n_{3} ; \varphi}^{\infty}+\operatorname{perm} .\left(n_{1}, n_{2}, n_{3}\right)\right)
\end{aligned}
$$

These coefficients can be given by the sum over the permutations of the corresponding gluon amplitude evaluated in $[42,47,60]$.

\section{$\mu^{2}$ Dependence Of The Bubble Coefficients}

For the double cut of the massive scalar loop multi-photon amplitude we choose the following basis for the loop momentum (see section 4.3 of [47])

$$
\bar{\ell}_{1}=y K_{1}^{b}+\frac{K_{1}^{2}(1-y)}{2\left(K_{1} \cdot \chi\right)} \chi+t\left|K_{1}^{b}\right\rangle\left[\chi_{1}\left|+\frac{y(1-y) K_{1}^{2}-\mu^{2}}{2 t\left(K_{1} \cdot \chi\right)}\right| \chi\right\rangle\left[K_{1}^{b} \mid,\right.
$$


with $K_{1}^{b}=K_{1}-\chi K_{1}^{2} /\left(2\left(K_{1} \cdot \chi\right)\right)$. The bubble coefficient has two components, a pure double cut term and a set of triangle subtraction terms:

$$
C_{2}^{[2]}=C_{2}^{\text {bub, }[2]}+\sum_{\left\{K_{3}\right\}} C_{2}^{\operatorname{tri}\left(K_{3}\right)[2]} .
$$

These components are expressed in terms of the large momentum scaling as,

$$
\begin{aligned}
C_{2}^{\text {bub }[2]} & =-i \inf _{\mu^{2}}\left[\left.\inf _{t}\left[\inf _{y}\left[A_{n_{1}}^{\text {tree }} A_{n_{2}}^{\text {tree }}\left(\bar{\ell}_{1}\left(y, t, \mu^{2}\right)\right]\right]\right]\right|_{\mu^{2}, t^{0}, y^{i} \rightarrow Y_{i}},\right. \\
C_{2}^{\text {tri }\left(K_{3}\right)[2]} & =-\frac{1}{2} \sum_{\sigma= \pm} \inf _{\mu^{2}}\left[\left.\inf _{t}\left[A_{n_{1}}^{\text {tree }} A_{n_{2}}^{\text {tree }} A_{n_{3}}^{\text {tree }}\left(\bar{\ell}_{1}^{\sigma}\left(y_{\sigma}, t, \mu^{2}\right)\right]\right]\right|_{\mu^{2}, t^{i} \rightarrow T_{i}} .\right.
\end{aligned}
$$

The non-vanishing integrals depend on $\mu^{2}$ and have the following large $\mu^{2}$ behaviour (see section 4.3 of [47] for detailed expressions)

$$
\begin{array}{rll}
\lim _{\mu^{2} \rightarrow \infty} Y_{0}=\mathcal{O}(1), & \lim _{\mu^{2} \rightarrow \infty} Y_{1}=\mathcal{O}(1), & \lim _{\mu^{2} \rightarrow \infty} Y_{2}=\mathcal{O}\left(\mu^{2}\right), \\
\lim _{\mu^{2} \rightarrow \infty} T_{1}=\mathcal{O}(1), & \lim _{\mu^{2} \rightarrow \infty} T_{2}=\mathcal{O}(1), & \lim _{\mu^{2} \rightarrow \infty} T_{3}=\mathcal{O}\left(\mu^{2}\right) .
\end{array}
$$

With an analysis similar to the one performed in section C.2 we obtain that the product of the tree amplitudes in (C.40) and (C.41) behaves as $O\left(\left(y^{2} / t\right)^{4-n}\right)$ therefore for $n>4$ external photons we have

$$
\inf _{t}\left[\inf _{y}\left[A_{n_{1}}^{\text {tree }} A_{n_{2}}^{\text {tree }}\left(\bar{\ell}_{1}\right)\right]\right]=0 .
$$

Hence there is no rational term contributions from the bubbles to the one-loop amplitude with $n>4$ external photons. For $n=5$ there is a priori a non vanishing contribution from the subtraction of triangles $C_{2}^{\text {tri }[K 3]}$ but as before these contributions vanish in the sum over all the orderings as required by Furry's theorem.

Both $C_{2}^{\text {bub[2] }}$ and $C_{3}^{\operatorname{tri}\left(K_{3}\right)[2]}$ for four external photons receive non-zero contributions which can be obtained by summing over the ordering of the corresponding gluon amplitude contributions which were evaluated in $[42,47,60]$.

Therefore there is a rational term contribution to the four point amplitude in agreement with the explicit computations performed in [38,70,71].

\section{The scalar box integral functions}

In this section we give a relation between the infra-red part of the massless scalar box integral functions evaluated in $D=4-2 \epsilon$ dimensions and triangle contributions. We will follow the notation of the refs. $[8,65]$.

We will use $k_{i}$ for massless legs $k_{i}^{2}=0$, and $K_{i}$ for massive legs $K_{i}^{2} \neq 0$. As well we will use $s_{i j}$ for either $-\left(k_{i}+k_{j}\right)^{2}$, or $-\left(k_{i}+K_{j}\right)^{2}$, or $-\left(K_{i}+K_{j}\right)^{2}$.

The infra-red divergent part of the massless scalar box integral function is given by

$$
\left.I_{4}\left(k_{1}, k_{2}, k_{3}, k_{4}\right)\right|_{I R}=r_{\Gamma} \frac{\mu^{2 \epsilon}}{s_{12} s_{23}} \frac{2}{\epsilon^{2}}\left(\left(-s_{12}\right)^{-\epsilon}+\left(-s_{23}\right)^{-\epsilon}\right) .
$$


The infra-red divergent part of the one-mass scalar box integral function is given by

$$
\left.I_{4}\left(k_{1}, k_{2}, k_{3}, K_{4}\right)\right|_{I R}=r_{\Gamma} \frac{\mu^{2 \epsilon}}{s_{12} s_{23}} \frac{2}{\epsilon^{2}}\left(\left(-s_{12}\right)^{-\epsilon}+\left(-s_{23}\right)^{-\epsilon}-\left(-K_{4}^{2}\right)^{-\epsilon}\right)
$$

The infra-red divergent part of the two-mass easy scalar box integral function is given by

$$
\left.I_{4}\left(k_{1}, K_{2}, k_{3}, K_{4}\right)\right|_{I R}=r_{\Gamma} \frac{\mu^{2 \epsilon}}{s_{12} s_{23}-K_{2}^{2} K_{4}^{2}} \frac{2}{\epsilon^{2}}\left(\left(-s_{12}\right)^{-\epsilon}+\left(-s_{23}\right)^{-\epsilon}-\left(-K_{2}^{2}\right)^{-\epsilon}-\left(-K_{4}^{2}\right)^{-\epsilon}\right)
$$

The infra-red divergent part of the two-mass hard scalar box integral function is given by

$$
\begin{aligned}
\left.I_{4}\left(k_{1}, k_{2}, K_{3}, K_{4}\right)\right|_{I R} & =r_{\Gamma} \frac{\mu^{2 \epsilon}}{s_{12} s_{23}} \frac{2}{\epsilon^{2}}\left(\left(-s_{12}\right)^{-\epsilon}+\left(-s_{23}\right)^{-\epsilon}-\left(-K_{3}^{2}\right)^{-\epsilon}-\left(-K_{4}^{2}\right)^{-\epsilon}\right) \\
& +r_{\Gamma} \frac{\mu^{2 \epsilon}}{s_{12} s_{23}} \frac{1}{\epsilon^{2}} \frac{\left(-K_{3}^{2}\right)^{-\epsilon}\left(-K_{4}^{2}\right)^{-\epsilon}}{\left(-s_{12}\right)^{-\epsilon}} \\
& =r_{\Gamma} \frac{\mu^{2 \epsilon}}{s_{12} s_{23}} \frac{1}{\epsilon^{2}}\left(\left(-s_{12}\right)^{-\epsilon}+2\left(-s_{23}\right)^{-\epsilon}-\left(-K_{3}^{2}\right)^{-\epsilon}-\left(-K_{4}^{2}\right)^{-\epsilon}\right) .
\end{aligned}
$$

The infra-red divergent part of the three-mass scalar box integral function is given by

$$
\begin{aligned}
\left.I_{4}\left(k_{1}, K_{2}, K_{3}, K_{4}\right)\right|_{I R} & =r_{\Gamma} \frac{\mu^{2 \epsilon}}{s_{12} s_{23}-K_{2}^{2} K_{4}^{2}} \frac{2}{\epsilon^{2}}\left(\left(-s_{12}\right)^{-\epsilon}+\left(-s_{23}\right)^{-\epsilon}\right) \\
& -r_{\Gamma} \frac{\mu^{2 \epsilon}}{s_{12} s_{23}-K_{2}^{2} K_{4}^{2}} \frac{2}{\epsilon^{2}}\left(\left(-K_{2}^{2}\right)^{-\epsilon}+\left(-K_{3}^{2}\right)^{-\epsilon}+\left(-K_{4}^{2}\right)^{-\epsilon}\right) \\
& +r_{\Gamma} \frac{\mu^{2 \epsilon}}{s_{12} s_{23}-K_{2}^{2} K_{4}^{2}} \frac{1}{\epsilon^{2}}\left(\frac{\left(-K_{2}^{2}\right)^{-\epsilon}\left(-K_{3}^{2}\right)^{-\epsilon}}{\left(-s_{23}\right)^{-\epsilon}}+\frac{\left(-K_{3}^{2}\right)^{-\epsilon}\left(-K_{4}^{2}\right)^{-\epsilon}}{\left(-s_{12}\right)^{-\epsilon}}\right) \\
& =r_{\Gamma} \frac{\mu^{2 \epsilon}}{s_{12} s_{23}-K_{2}^{2} K_{4}^{2}} \frac{1}{\epsilon^{2}}\left(\left(-s_{12}\right)^{-\epsilon}+\left(-s_{23}\right)^{-\epsilon}-\left(-K_{2}^{2}\right)^{-\epsilon}-\left(-K_{4}^{2}\right)^{-\epsilon}\right),
\end{aligned}
$$

where $r_{\Gamma}=\Gamma(1+\epsilon) \Gamma(1-\epsilon)^{2} / \Gamma(1-2 \epsilon)$.

The divergent dimensionless one-mass and two-mass scalar triangle functions are given by

$$
\begin{aligned}
& \tilde{I}_{3}\left(k_{1}, k_{2}, K_{3}\right) \equiv\left(-K_{3}^{2}\right) I_{3}\left(k_{1}, k_{2}, K_{3}\right)=r_{\Gamma} \frac{\mu^{2 \epsilon}}{\epsilon^{2}}\left(-K_{3}^{2}\right)^{-\epsilon} \\
& \tilde{I}_{3}\left(k_{1}, K_{2}, K_{3}\right) \equiv\left(K_{2}^{2}-K_{3}^{2}\right) I_{3}\left(k_{1}, k_{2}, K_{3}\right)=r_{\Gamma} \frac{\mu^{2 \epsilon}}{\epsilon^{2}}\left(\left(-K_{2}^{2}\right)^{-\epsilon}-\left(-K_{3}^{2}\right)^{-\epsilon}\right) .
\end{aligned}
$$

These expressions imply that all the divergent parts of the dimensionless scalar box integral functions can be expressed as linear combination of the infra-red parts of the dimensionless 
scalar triangle functions in the following way

$$
\begin{aligned}
\left.\tilde{I}_{4}\left(k_{1}, k_{2}, k_{3}, k_{4}\right)\right|_{I R} & =\left.2\left(\tilde{I}_{3}\left(k_{3}, k_{4}, k_{1}+k_{2}\right)+\tilde{I}_{3}\left(k_{1}, k_{4}, k_{2}+k_{3}\right)\right)\right|_{I R} \\
\left.\tilde{I}_{4}\left(k_{1}, k_{2}, k_{3}, K_{4}\right)\right|_{I R} & =\left(\tilde{I}_{3}\left(k_{1}, k_{2}, k_{3}+K_{4}\right)+\tilde{I}_{3}\left(k_{2}, k_{3}, k_{1}+K_{4}\right)\right. \\
& \left.+\tilde{I}_{3}\left(k_{3}, k_{1}+k_{2}, K_{4}\right)+\tilde{I}_{3}\left(k_{1}, k_{2}+k_{3}, K_{4}\right)\right)\left.\right|_{I R} \\
\left.\tilde{I}_{4}\left(k_{1}, K_{2}, k_{3}, K_{4}\right)\right|_{I R} & =\left.2\left(\tilde{I}_{3}\left(k_{1}, K_{2}, k_{3}+K_{4}\right)+\tilde{I}_{3}\left(k_{1}, k_{3}+K_{2}, K_{4}\right)\right)\right|_{I R} \\
\left.\tilde{I}_{4}\left(k_{1}, k_{2}, K_{3}, K_{4}\right)\right|_{I R} & =\left(\tilde{I}_{3}\left(k_{1}, k_{2}, K_{3}+K_{4}\right)+\tilde{I}_{3}\left(k_{1}, k_{2}+K_{3}, K_{4}\right)\right. \\
& \left.+\tilde{I}_{3}\left(k_{2}, K_{3}, k_{1}+K_{4}\right)\right)\left.\right|_{I R} \\
\left.\tilde{I}_{4}\left(k_{1}, K_{2}, K_{3}, K_{4}\right)\right|_{I R} & =\left.\left(\tilde{I}_{3}\left(k_{1}, K_{2}, K_{3}+K_{4}\right)+\tilde{I}_{3}\left(k_{1}, K_{2}+K_{3}, K_{4}\right)\right)\right|_{I R}
\end{aligned}
$$

\section{References}

[1] Z. Bern, L. J. Dixon and D. A. Kosower, "On-Shell Methods in Perturbative QCD," Annals Phys. 322 (2007) 1587 [arXiv:0704.2798 [hep-ph]].

[2] Z. Bern, L. J. Dixon, M. Perelstein and J. S. Rozowsky, "Multi-leg one-loop gravity amplitudes from gauge theory," Nucl. Phys. B 546, 423 (1999) [arXiv:hep-th/9811140].

[3] Z. Bern, N. E. J. Bjerrum-Bohr and D. C. Dunbar, "Inherited twistor-space structure of gravity loop amplitudes," JHEP 0505, 056 (2005) [arXiv:hep-th/0501137].

[4] N. E. J. Bjerrum-Bohr, D. C. Dunbar and H. Ita, "Six-point one-loop N = 8 supergravity NMHV amplitudes and their IR behaviour," Phys. Lett. B 621, 183 (2005) [arXiv:hep-th/0503102].

[5] N. E. J. Bjerrum-Bohr, D. C. Dunbar, H. Ita, W. B. Perkins and K. Risager, "MHV-vertices for gravity amplitudes," JHEP 0601, 009 (2006) [arXiv:hep-th/0509016].

[6] N. E. J. Bjerrum-Bohr, D. C. Dunbar, H. Ita, W. B. Perkins and K. Risager, "The no-triangle hypothesis for $\mathrm{N}=8$ supergravity," JHEP 0612 (2006) 072 [arXiv:hep-th/0610043].

[7] Z. Bern, J. J. Carrasco, D. Forde, H. Ita and H. Johansson, "Unexpected Cancellations in Gravity Theories," Phys. Rev. D 77 (2008) 025010 [arXiv:0707.1035 [hep-th]].

[8] Z. Bern, L. J. Dixon and D. A. Kosower, "Dimensionally Regulated One Loop Integrals," Phys. Lett. B 302 (1993) 299 [Erratum-ibid. B 318 (1993) 649] [arXiv:hep-ph/9212308]; "Dimensionally regulated pentagon integrals," Nucl. Phys. B 412, 751 (1994) [arXiv:hep-ph/9306240].

[9] Z. Bern, L. J. Dixon, D. C. Dunbar and D. A. Kosower, "One loop n point gauge theory amplitudes, unitarity and collinear limits," Nucl. Phys. B 425 (1994) 217 [arXiv:hep-ph/9403226].

[10] L. M. Brown and R. P. Feynman, "Radiative corrections to Compton scattering," Phys. Rev. 85 (1952) 231. G. Passarino and M. J. G. Veltman, "One Loop Corrections For $e^{+} e^{-}$ Annihilation Into $\mu^{+} \mu^{-}$In The Weinberg Model," Nucl. Phys. B 160 (1979) 151.

[11] J. M. Campbell, E. W. N. Glover and D. J. Miller, "One-loop tensor integrals in dimensional regularisation," Nucl. Phys. B 498 (1997) 397 [arXiv:hep-ph/9612413]. 
[12] A. Denner and S. Dittmaier, "Reduction schemes for one-loop tensor integrals," Nucl. Phys. B 734 (2006) 62 [arXiv:hep-ph/0509141].

[13] Z. Bern, L. J. Dixon, D. A. Kosower, R. Roiban, M. Spradlin, C. Vergu and A. Volovich, "The Two-Loop Six-Gluon MHV Amplitude in Maximally Supersymmetric Yang-Mills Theory," Phys. Rev. D 78 (2008) 045007 [arXiv:0803.1465 [hep-th]].

[14] F. Cachazo, "Sharpening The Leading Singularity," arXiv:0803.1988 [hep-th].

[15] F. Cachazo, M. Spradlin and A. Volovich, "Leading Singularities of the Two-Loop Six-Particle MHV Amplitude," arXiv:0805.4832 [hep-th].

[16] M. Spradlin, A. Volovich and C. Wen, "Three-Loop Leading Singularities and BDS Ansatz for Five Particles," arXiv:0808.1054 [hep-th].

[17] N. Berkovits, "New higher-derivative R**4 theorems," Phys. Rev. Lett. 98 (2007) 211601 [arXiv:hep-th/0609006].

[18] M. B. Green, J. G. Russo and P. Vanhove, "Non-renormalisation conditions in type II string theory and maximal supergravity," JHEP 0702, 099 (2007) [arXiv:hep-th/0610299].

[19] M. B. Green, J. G. Russo and P. Vanhove, "Ultraviolet properties of maximal supergravity," Phys. Rev. Lett. 98, 131602 (2007) [arXiv:hep-th/0611273].

[20] Z. Bern, L. J. Dixon and R. Roiban, "Is N = 8 supergravity ultraviolet finite?," Phys. Lett. B 644 (2007) 265 [arXiv:hep-th/0611086].

[21] Z. Bern, J. J. Carrasco, L. J. Dixon, H. Johansson, D. A. Kosower and R. Roiban, "Three-Loop Superfiniteness of N=8 Supergravity," Phys. Rev. Lett. 98 (2007) 161303 [arXiv:hep-th/0702112].

[22] Z. Bern, J. J. M. Carrasco, L. J. Dixon, H. Johansson and R. Roiban, "Manifest Ultraviolet Behavior for the Three-Loop Four-Point Amplitude of N=8 Supergravity," arXiv:0808.4112 [hep-th].

[23] N. E. J. Bjerrum-Bohr and P. Vanhove, "Absence of Triangles in Maximal Supergravity Amplitudes," JHEP 0810 (2008) 006 [arXiv:0805.3682 [hep-th]].

[24] N. E. J. Bjerrum-Bohr and P. Vanhove, "Explicit Cancellation of Triangles in One-loop Gravity Amplitudes," JHEP 0804 (2008) 065 [arXiv:0802.0868 [hep-th]].

[25] N. Arkani-Hamed, F. Cachazo and J. Kaplan, "What is the Simplest Quantum Field Theory?," arXiv:0808.1446 [hep-th].

[26] Z. Bern and D. A. Kosower, "The Computation of loop amplitudes in gauge theories," Nucl. Phys. B 379 (1992) 451.

[27] Z. Bern and D. C. Dunbar, "A Mapping between Feynman and string motivated one loop rules in gauge theories," Nucl. Phys. B 379 (1992) 562.

[28] M. J. Strassler, "Field theory without Feynman diagrams: One loop effective actions," Nucl. Phys. B 385 (1992) 145 [arXiv:hep-ph/9205205].

[29] Z. Bern, "String Based Perturbative Methods For Gauge Theories," arXiv:hep-ph/9304249.

[30] C. Schubert, "Perturbative quantum field theory in the string-inspired formalism," Phys. Rept. 355 (2001) 73 [arXiv:hep-th/0101036]. 
[31] M. G. Schmidt and C. Schubert, "Worldline Green functions for multiloop diagrams," Phys. Lett. B 331 (1994) 69 [arXiv:hep-th/9403158].

[32] K. Roland and H. T. Sato, "Multiloop World-Line Green Functions from String Theory," Nucl. Phys. B 480 (1996) 99 [arXiv:hep-th/9604152].

[33] P. Dai and W. Siegel, "Worldline green functions for arbitrary Feynman diagrams," Nucl. Phys. B 770 (2007) 107 [arXiv:hep-th/0608062].

"Worldgraph Approach to Yang-Mills Amplitudes from N=2 Spinning Particle," arXiv:0807.0391 [hep-th].

[34] M. B. Green, J. G. Russo and P. Vanhove, "Modular properties of two-loop maximal supergravity and connections with string theory," JHEP 0807 (2008) 126 [arXiv:0807.0389 [hep-th]].

[35] Z. Bern, L. J. Dixon, D. C. Dunbar, M. Perelstein and J. S. Rozowsky, "On the relationship between Yang-Mills theory and gravity and its implication for ultraviolet divergences," Nucl. Phys. B 530, 401 (1998) [arXiv:hep-th/9802162].

[36] T. Binoth, G. Heinrich, T. Gehrmann and P. Mastrolia, "Six-Photon Amplitudes," Phys. Lett. B 649 (2007) 422 [arXiv:hep-ph/0703311].

[37] C. Bernicot and J. P. Guillet, "Six-Photon Amplitudes in Scalar QED," JHEP 0801 (2008) 059 [arXiv:0711.4713 [hep-ph]].

[38] G. Mahlon, "One loop multi - photon helicity amplitudes," Phys. Rev. D 49 (1994) 2197 [arXiv:hep-ph/9311213].

[39] N. E. J. Bjerrum-Bohr and P. Vanhove, "On Cancellations of Ultraviolet Divergences in Supergravity Amplitudes," arXiv:0806.1726 [hep-th].

[40] R. Britto, F. Cachazo and B. Feng, "Generalized unitarity and one-loop amplitudes in N = 4 super-Yang-Mills," Nucl. Phys. B 725 (2005) 275 [arXiv:hep-th/0412103].

[41] C. Anastasiou, R. Britto, B. Feng, Z. Kunszt and P. Mastrolia, "Unitarity cuts and reduction to master integrals in d dimensions for one-loop amplitudes," JHEP 0703, 111 (2007) [arXiv:hep-ph/0612277]. "D-dimensional unitarity cut method," Phys. Lett. B 645, 213 (2007) [arXiv:hep-ph/0609191].

[42] R. Britto, B. Feng and P. Mastrolia, 'Closed-Form Decomposition of One-Loop Massive Amplitudes," Phys. Rev. D 78 (2008) 025031 [arXiv:0803.1989 [hep-ph]]. R. Britto, B. Feng and G. Yang, "Complete One-Loop Amplitudes With Massless Propagators," JHEP 0809, 089 (2008) [arXiv:0803.3147 [hep-ph]]. B. Feng and G. Yang, "Unitarity Method with Spurious Pole," arXiv:0806.4016 [hep-ph].

[43] D. Forde, "Direct extraction of one-loop integral coefficients," Phys. Rev. D 75 (2007) 125019 [arXiv:0704.1835 [hep-ph]].

[44] G. Ossola, C. G. Papadopoulos and R. Pittau, "Reducing full one-loop amplitudes to scalar integrals at the integrand level," Nucl. Phys. B 763 (2007) 147 [arXiv:hep-ph/0609007]. "On the Rational Terms of the one-loop amplitudes," JHEP 0805 (2008) 004 [arXiv:0802.1876 [hep-ph]].

[45] W. T. Giele, Z. Kunszt and K. Melnikov, "Full one-loop amplitudes from tree amplitudes," JHEP 0804 (2008) 049 [arXiv:0801.2237 [hep-ph]]. 
[46] C. F. Berger et al., "An Automated Implementation of On-Shell Methods for One-Loop Amplitudes," Phys. Rev. D 78 (2008) 036003 [arXiv:0803.4180 [hep-ph]].

[47] S. D. Badger, "Direct Extraction Of One-Loop Rational Terms," arXiv:0806.4600 [hep-ph].

[48] L. Brink, P. Di Vecchia and P. S. Howe, "A Locally Supersymmetric And Reparametrization Invariant Action For The Spinning String," Phys. Lett. B 65 (1976) 471.

"A Lagrangian Formulation Of The Classical And Quantum Dynamics Of Spinning Particles," Nucl. Phys. B 118 (1977) 76.

[49] Z. Bern, L. J. Dixon, D. C. Dunbar and D. A. Kosower, "Fusing gauge theory tree amplitudes into loop amplitudes," Nucl. Phys. B 435 (1995) 59 [arXiv:hep-ph/9409265].

[50] R. K. Ellis, W. T. Giele, Z. Kunszt and K. Melnikov, "Masses, fermions and generalized D-dimensional unitarity," arXiv:0806.3467 [hep-ph].

[51] M. E. Peskin and D. V. Schroeder, "An Introduction To Quantum Field Theory," Reading, USA: Addison-Wesley (1995) $842 \mathrm{p}$.

[52] R. Kleiss and W. J. Stirling, "Cross-Sections For The Production Of An Arbitrary Number Of Photons In Electron - Positron Annihilation," Phys. Lett. B 179 (1986) 159.

[53] M. T. Grisaru, H. N. Pendleton and P. van Nieuwenhuizen, "Supergravity And The S Matrix," Phys. Rev. D 15, 996 (1977).

M. T. Grisaru and H. N. Pendleton, "Some Properties Of Scattering Amplitudes In Supersymmetric Theories," Nucl. Phys. B 124 (1977) 81.

[54] S. J. Parke and T. R. Taylor, "An Amplitude for $n$ Gluon Scattering," Phys. Rev. Lett. 56, 2459 (1986).

[55] F. A. Berends and W. T. Giele, "Recursive Calculations for Processes with n Gluons," Nucl. Phys. B 306 (1988) 759.

"Multiple Soft Gluon Radiation in Parton Processes," Nucl. Phys. B 313 (1989) 595.

[56] M. L. Mangano and S. J. Parke, "Multiparton amplitudes in gauge theories," Phys. Rept. 200 (1991) 301 [arXiv:hep-th/0509223].

[57] K. J. Ozeren and W. J. Stirling, "MHV techniques for QED processes," JHEP 0511 (2005) 016 [arXiv:hep-th/0509063].

[58] F. Cachazo, P. Svrcek and E. Witten, "MHV vertices and tree amplitudes in gauge theory," JHEP 0409 (2004) 006 [arXiv:hep-th/0403047].

[59] R. Britto, F. Cachazo and B. Feng, "New recursion relations for tree amplitudes of gluons," Nucl. Phys. B 715 (2005) 499 [arXiv:hep-th/0412308].

R. Britto, F. Cachazo, B. Feng and E. Witten, "Direct proof of tree-level recursion relation in Yang-Mills theory," Phys. Rev. Lett. 94 (2005) 181602 [arXiv:hep-th/0501052].

[60] Z. Bern and A. G. Morgan, "Massive Loop Amplitudes from Unitarity," Nucl. Phys. B 467 (1996) 479 [arXiv:hep-ph/9511336].

[61] Z. Bern, L. J. Dixon, D. C. Dunbar and D. A. Kosower, "One-loop self-dual and N =4 superYang-Mills," Phys. Lett. B 394, 105 (1997) [arXiv:hep-th/9611127].

[62] C. Schwinn and S. Weinzierl, "On-shell recursion relations for all Born QCD amplitudes," JHEP 0704 (2007) 072 [arXiv:hep-ph/0703021]. 
[63] P. Benincasa, C. Boucher-Veronneau and F. Cachazo, "Taming tree amplitudes in general relativity," JHEP 0711 (2007) 057 [arXiv:hep-th/0702032].

[64] N. Arkani-Hamed and J. Kaplan, "On Tree Amplitudes in Gauge Theory and Gravity," JHEP 0804 (2008) 076 [arXiv:0801.2385 [hep-th]].

[65] R. K. Ellis and G. Zanderighi, "Scalar one-loop integrals for QCD," JHEP 0802 (2008) 002 [arXiv:0712.1851 [hep-ph]].

A repostitory of one-loop integrals is available at http://qcdloop.fnal.gov .

[66] G. Mahlon, "Use of recursion relations to compute one loop helicity amplitudes," arXiv:hep-ph/9412350.

[67] L. F. Alday and J. M. Maldacena, "Gluon scattering amplitudes at strong coupling," JHEP 0706 (2007) 064 [arXiv:0705.0303 [hep-th]].

[68] J. M. Drummond, G. P. Korchemsky and E. Sokatchev, "Conformal properties of four-gluon planar amplitudes and Wilson loops," Nucl. Phys. B 795 (2008) 385 [arXiv:0707.0243 [hep-th]].

[69] W. T. Giele and E. W. N. Glover, "Higher Order Corrections To Jet Cross-Sections In E+ EAnnihilation," Phys. Rev. D 46 (1992) 1980.

[70] G. Jikia and A. Tkabladze, "Photon-Photon Scattering At The Photon Linear Collider," Phys. Lett. B 323 (1994) 453 [arXiv:hep-ph/9312228].

[71] G. J. Gounaris, P. I. Porfyriadis and F. M. Renard, "The gamma gamma -> gamma gamma process in the standard and SUSY models at high energies," Eur. Phys. J. C 9 (1999) 673 [arXiv:hep-ph/9902230].

[72] D. C. Dunbar and P. S. Norridge, "Infinities within graviton scattering amplitudes," Class. Quant. Grav. 14 (1997) 351 [arXiv:hep-th/9512084].

[73] M. B. Green, J. H. Schwarz and L. Brink, "N=4 Yang-Mills And N=8 Supergravity As Limits Of String Theories," Nucl. Phys. B 198, 474 (1982).

[74] S. D. Badger, E. W. N. Glover, V. V. Khoze and P. Svrcek, "Recursion relations for gauge theory amplitudes with massive particles," JHEP 0507 (2005) 025 [arXiv:hep-th/0504159].

[75] D. Forde and D. A. Kosower, "All-multiplicity amplitudes with massive scalars," Phys. Rev. D 73 (2006) 065007 [arXiv:hep-th/0507292].

[76] A. Brandhuber, S. McNamara, B. J. Spence and G. Travaglini, "Loop amplitudes in pure Yang-Mills from generalised unitarity," JHEP 0510 (2005) 011 [arXiv:hep-th/0506068].

[77] C. F. Berger, Z. Bern, L. J. Dixon, D. Forde and D. A. Kosower, "Bootstrapping one-loop QCD amplitudes with general helicities," Phys. Rev. D 74 (2006) 036009 [arXiv:hep-ph/0604195]. 